\title{
The Revaluation of Plant-Derived Terpenes to Fight Antibiotic-Resistant Infections
}

\author{
Floriana Cappiello ${ }^{1, \dagger}$, Maria Rosa Loffredo ${ }^{1,+}$, Cristina Del Plato ${ }^{2,3}$, Silvia Cammarone ${ }^{2}$, \\ Bruno Casciaro $^{3, *}{ }^{\circledR}$, Deborah Quaglio ${ }^{2, *}$, Maria Luisa Mangoni ${ }^{1}\left(\mathbb{D}\right.$, Bruno Botta $^{2}(\mathbb{D}$ and \\ Francesca Ghirga ${ }^{3}$ \\ 1 Laboratory affiliated to Pasteur Italia-Fondazione Cenci Bolognetti, Department of Biochemical Sciences, \\ Sapienza University of Rome, P.le Aldo Moro 5, 00185 Rome, Italy; floriana.cappiello@uniroma1.it (F.C.); \\ mariarosa.loffredo@uniroma1.it (M.R.L.); marialuisa.mangoni@uniroma1.it (M.L.M.) \\ 2 Department of Chemistry and Technology of Drugs, "Department of Excellence 2018-2022", \\ Sapienza University of Rome, P.le Aldo Moro 5, 00185 Rome, Italy; cristina.delplato@uniroma1.it (C.D.P.); \\ silvia.cammarone@uniroma1.it (S.C.); bruno.botta@uniroma1.it (B.B.) \\ 3 Center For Life Nano Science@Sapienza, Istituto Italiano di Tecnologia, Viale Regina Elena 291, \\ 00161 Rome, Italy; francesca.ghirga@iit.it \\ * Correspondence: bruno.casciaro@iit.it (B.C.); deborah.quaglio@uniroma1.it (D.Q.) \\ + These authors equally contributed to the work.
}

Received: 18 May 2020; Accepted: 10 June 2020; Published: 13 June 2020

\begin{abstract}
The discovery of antibiotics has revolutionized the medicine and treatment of microbial infections. However, the current scenario has highlighted the difficulties in marketing new antibiotics and an exponential increase in the appearance of resistant strains. On the other hand, research in the field of drug-discovery has revaluated the potential of natural products as a unique source for new biologically active molecules and scaffolds for the medicinal chemistry. In this review, we first contextualized the worldwide problem of antibiotic resistance and the importance that natural products of plant origin acquire as a source of new lead compounds. We then focused on terpenes and their potential development as antimicrobials, highlighting those studies that showed an activity against conventional antibiotic-resistant strains.
\end{abstract}

Keywords: antibiotic resistance; plant secondary metabolites; plant-derived natural products; terpenes; diterpenes; triterpenes; multidrug-resistant strains.

\section{Introduction}

The discovery of penicillin by Alexander Fleming (1881-1955) drastically revolutionized modern medicine and represented the beginning of the antibiotic era that saved millions of lives from bacterial infections [1]. Since then, according to the definition expressed by Selman Waksman, any small molecule, produced by a microbe, with antagonistic properties on the growth of other microbes was defined as "antibiotic" [2]. During the "Antibiotic Golden Age" (1940-1970), hundreds of molecules were isolated, characterized, classified and marketed [3-5]. For decades, antibiotics were used as "wonder drugs" to combat microbes but also as prophylactic agents in the field of agriculture, fish industry and breeding. The massive use, in addition to a high rate of prescriptions, mismanagement in the form of self-medication or interruption of therapy, led to an exponential appearance of resistant bacterial infections and consequent failure of antibiotic therapies [6,7]. This was followed by a post-antibiotic era where the discovery of new compounds is still in sharp decline, together with a proportional rise in the appearance of resistant strains [8-12]. The problem of antibiotic resistance is crucial and scientific research should not lag behind. Only in Europe, in fact, about 700 thousand cases of antibiotic-resistant infections provoke 33,000 deaths every year, with an estimated cost above 
$€ 1.5$ billion [13]. However, it should be considered that an estimated $\$ 200$ million is required for a molecule to reach commercialization, with the possible risk of a rapid development of resistance and consequent interruption of clinical development [14]. Despite this, it is evident that new molecules with antimicrobial properties are urgently needed and nature represents the primary source of biologically active compounds, in agreement with Fleming's statement: "I did not invent penicillin. Nature did that. I only discovered it by accident" [15]. In this review, we focus on the relevance of natural compounds from plants (plant-derived natural products, PDNPs) in the 21st century drug discovery, with emphasis to terpenes showing antimicrobial activities and that can represent promising candidates to fight multidrug-resistant (MDR) microbial infections.

\section{Antimicrobial Resistance}

Biologically active molecules are defined as antimicrobials if they are able to inhibit growth or to kill certain or various classes of microorganisms. However, microorganisms have developed several mechanisms to circumvent the action of these antimicrobial compounds. In this context, it is worthwhile to specify the difference between antimicrobial resistance and persistence. Resistance to a given molecule is maintained from the mother cell to the daughter cells, unless mutations make them susceptible again [16]. In comparison, persistence is defined as the ability of microbial cells to be recalcitrant to the antibiotic action, as they enter into a stationary phase of their growth (dormant cells). This leads to the inefficacy of the antibiotic agents since most of them act by inhibiting or interacting with specific metabolic processes that are not active in dormant cells [17,18]. It is also important to define the two major types of antimicrobial resistance: natural and acquired [19,20]. Natural resistance can be constantly expressed in the bacterial species (intrinsic resistance), whereas acquired resistance is expressed only upon exposure to an antimicrobial agent (induced resistance) [21]. The reduced permeability of the outer membrane and the activity of efflux pumps are classic examples of intrinsic resistance [22]. Acquired resistance occurs through acquisition of genetic material by means of transformation, conjugation, transposition (horizontal gene transfer) or by mutation in the chromosomal DNA $[23,24]$. Mutation of the drug target or in those genes involved in the regulation of drug transporters are examples of acquired resistance [19].

\section{Mechanisms of Antimicrobial Resistance}

Gram(+) and Gram(-) bacteria possess and/or have developed several mechanisms of antimicrobial resistance that fall into five major categories (Figure 1):

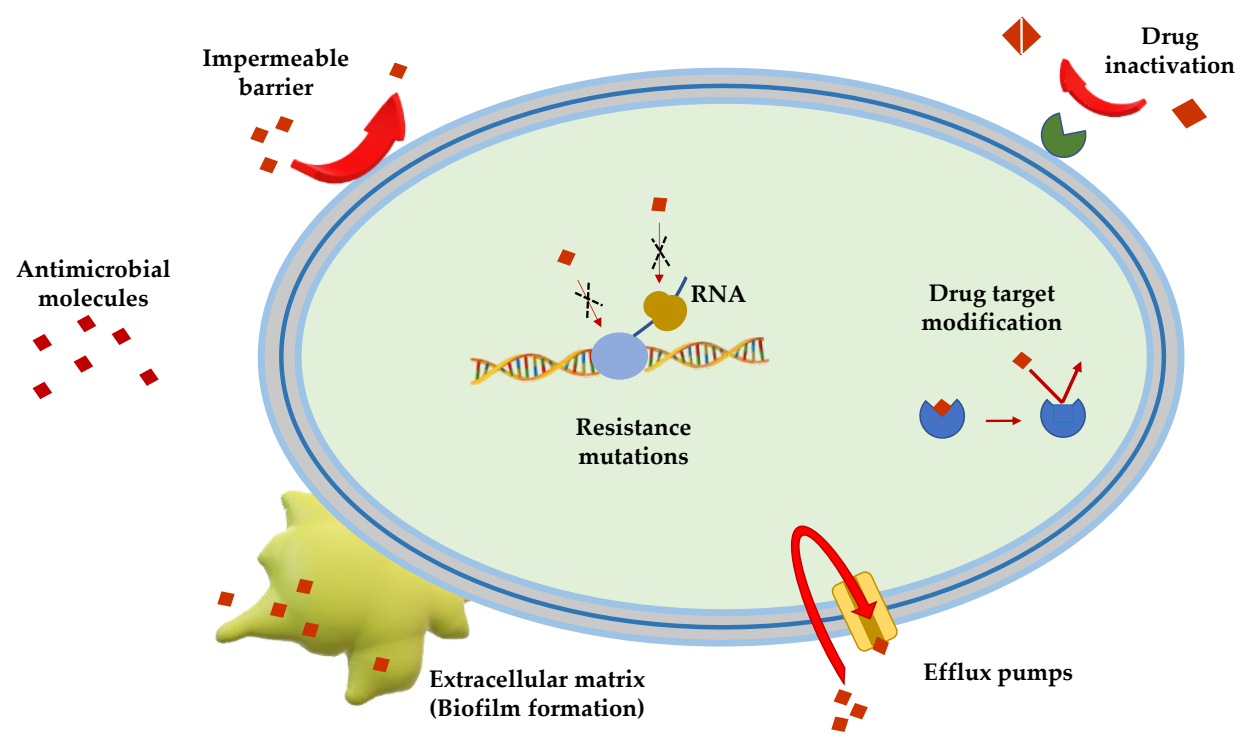

Figure 1. Schematic representation of the principal mechanisms of antibiotic resistance. 
Limitation of drug uptake. Bacteria can be intrinsically resistant to a certain antimicrobial due to their structure and morphology. Lipopolysaccharide (LPS) in Gram(-) bacteria, for example, provides a physical barrier that protects the cell from several groups of large molecules [25]. In these bacteria, drugs are internalized through porin channels that generally allow the uptake of hydrophilic molecules. Mutations that change their selectivity or that reduce the number of expressed porins are the two major mechanisms of antimicrobial resistance $[26,27]$. Gram(+) bacteria, lacking outer membrane, possess a peptidoglycan cell wall and the restricting drug uptake is not as prevalent. However, pathogenic bacterial species, i.e., Staphylococcus aureus, have developed a mechanism which consists in thickening the cell wall to limit the amount of drug that enters the cell [28,29]. Mycoplasma spp., devoid of cell wall, are intrinsically resistant to antimicrobials (e.g., $\beta$-lactams and glycopeptides) that interfere with cell wall synthesis and regulation [30].

Drug inactivation. Bacteria can produce several enzymes or molecules that inactivate drugs by covalent binding or enzymatic processes. Firstly, common antibiotics (e.g., aminoglycosides, streptogramins, fluoroquinolones, chloramphenicol) could be inactivated by acetylation, phosphorylation or adenylation; secondly, hydrolyzation is the primary mechanism by which bacteria can inactivate $\beta$-lactam antibiotics (e.g., cephalosporins, penicillins and cephamycins). $\beta$-lactamases are the most common example: these enzymes provide resistance to $\beta$-lactam antibiotics by hydrolyzing a specific site in the $\beta$-lactam ring structure [31]. Recently, $\beta$-lactamases were found to be active against carbapenems in Enterobacteriaceae (carbapenemases, i.e., Klebsiella pneumoniae carbapenemases and carbapenem-resistant enterobacteriaceae enzymes) [31].

Mutation/alteration of the drug target. The majority of antimicrobials have a specific mechanism of action against a specific cellular target and this is one of the reasons why bacteria are not susceptible to a certain class of molecules [32]. Gram(+) strains, for instance, become resistant to $\beta$-lactam drugs via alteration of the penicillin-binding proteins, that are transpeptidases involved in the cell wall construction [33]. S. aureus acquires resistance to the glycopeptide vancomycin by decreasing the binding ability of this molecule to the cell wall, as a consequence of a modification of the terminal d-Ala-d-Ala moiety of the peptidoglycan precursor lipid II [34].

Drug efflux. Bacteria can eliminate internalized toxic substances through a mechanism involving efflux pumps, which can be constitutively expressed or overexpressed under certain conditions. Many of these pumps have the capability to transport different types of substances. They are properly named multi-drug efflux pumps [35] and their increased number is generally associated to high-level of resistance to clinically significant bacterial infections [36,37].

Biofilm formation. In conditions of environmental stress, scarcity of nutrients, presence of antimicrobial molecules, some bacterial species can switch from a motile to a sessile lifestyle, named biofilm. This is a bacterial community able to colonize abiotic (e.g., medical devices and implants [38-40]) and biotic surfaces (e.g., human tissues [41-43]). Biofilm formation is a strategy used by pathogenic bacteria to protect themselves from the external stressful conditions by producing a thick and sticky extracellular matrix which contains DNA, proteins and polysaccharides. In addition, biofilm cells enter into a slow division rate, which weakens the effect of antibiotic molecules targeting specific cellular processes. Thus, biofilms are often associated to chronic infections and molecules capable to disrupt these communities and/or to inhibit their formation are highly demanded [44,45].

\section{Plant Derived Natural Products}

The practice of using plants for medicinal purposes is thousands of years old. Since the earliest civilizations, plants have played a major role in medicine due to their variability and abundance of therapeutic agents $[46,47]$. Notably, the first manuscript that reports the use of medicinal plants dates back to $2600 \mathrm{BC}$; it describes a complex medical system, with the use of over 1000 medicinal plants in Mesopotamia [48]. The "Ebers Papyrus" from about 1500 BC describes the usage of about 700 drugs (mainly plant-derived) in the Egyptian medicine, while traditional Chinese medicine has been extensively documented and nowadays it is still of reference [48-50]. In the West, the tradition of 
medicinal plants has foundations in the culture of Greek and Roman civilizations, as proved by the compendiums written by Dioscorides and Pliny the Elder, respectively [51]. However, until the 18th century $\mathrm{AD}$, the use of plants in medicine has been based on empirical evidence. It was only with the first studies conducted by Anton von Störck on poisonous herbs (i.e., colchicum and aconite) and by William Withering on foxglove, that the foundations of a scientific research on medicinal plants were laid [52]. Rational drug discovery began in 1806 when Friedrich Sertürner isolated a bioactive compound from the poppy plant, i.e., the alkaloid with analgesic and sleep-inducing effects and that he called "morphine" in honor of the Greek God of dreams, Morpheus. This induced a significant boost in the research and isolation of bioactive compounds from plants, as evidenced by the numerous discoveries of the following decades. In 1820, Runge isolated caffeine from Coffea arabica; in 1824 the anti-tussive agent codeine was isolated from poppy by Robiquet, while in 1848 the anti-spasmodic alkaloid papaverine was identified by Merck Fraz; in 1869, digoxin (digitalis) was isolated from Digitalis lanata by Nativelle [53]. These discoveries led to an exponential growth of scientific and economic interest in the PDNPs which reaches up to the present day: $\sim 25 \%$ of the estimated 1.1 trillion US dollars invested in the pharmaceutical market annually, come from PDNPs [53]. Thanks to computational and biological approaches it has been easier to proceed with the identification, selection and production of PDNPs which nowadays possess a renewed scientific and economic potential [54,55].

\section{Plant-Derived Drug Discovery: The Two Sides of the Coin}

Plant-derived drug discovery is currently hindered by a series of scientific, social and economic factors that deserve to be analyzed in order to contextualize the actual scenario of the pharmaceutical market.

Socio-economic factors. A main issue here is the access to starting materials. Initially, there is a process of correct identification and nomenclature that cannot be automated and requires experts in the field. These experts are also necessary for the correct collection, documentation and preparation of the investigated herbarium, but they are rather rare [56,57]. The isolation processes, albeit with high yield, lead to very low quantities of active compounds, thus requiring a very high amount of starting material. This is a crucial factor; many plants need precise habitats for their growth and optimal production of metabolites and have seasonal life cycles, making their harvest difficult to scale-up. Furthermore, the ecological balance of the belonging niche must be taken into consideration: in fact, it is important to evaluate the impact that a reduction in the number of plants in the niche can have on food chains and other living species. Finally, many plants grow only in certain regions and political factors must be taken into account for their harvest, such as wars, international relations between states, import/export laws [56].

Scientific factors. One of the most relevant concerns is undoubtedly the quality of the raw material. This is influenced by various conditions, including the time of harvest, the quality of the soil, possible environmental contamination as well as the type of processing and storage [58]. Biologically active compounds often have a complex chemical structure, with numerous chiral centers and substituents containing oxygen, which make their synthesis difficult in the laboratory. The extracts obtained from natural samples have a high viscosity, can precipitate or aggregate easily, but, above all, they often consist of a mixture of bioactive compounds that makes biological activity tests of dubious interpretation [59-63]. Another important issue is the mechanism of action that is not well defined yet, for some common compounds [64].

Besides the challenges mentioned above, PDNPs are considered promising candidates for the development of new drugs, thanks to their intrinsic characteristics.

First, they represent a direct source of therapeutic agents or phytomedicines. Between 1981 and 2014, the Food and Drug Administration (FDA) approved 1562 new drugs from natural sources, of which $141(9 \%)$ were botanical mixtures and $64(4 \%)$ were unaltered natural products [53]. According to recent analysis [49,53], the percentage of pharmacologically and phytochemically investigated plants is around $6 \%$ and $15 \%$ of the existing plant species, respectively. The plant kingdom can therefore 
be considered to be not fully explored and holds enormous potential. Furthermore, for some plants there is a well-documented millennial tradition of ethnopharmacology which represents an excellent starting point for more accurate scientific studies [65,66]. In addition, the advent of new techniques in separation, purification and characterization of novel compounds significantly improved the efficiency of these processes and, today, an important challenge is the generation of high-quality libraries of natural products that might allow the fast identification of lead compounds for drug discovery progression [67-75]. Importantly, PDNPs serve as scaffold for the synthesis of libraries with several chemical structures and for the design of lead compounds with a desired biological activity or markers for a specific detection [76-78]. These objectives can be achieved thanks to the contingent biological and computational approaches that can be used following one of these proposed models: the forward pharmacology or reverse pharmacology model. In the first case, the evidence of biological activity in vivo (i.e., antimicrobial assays, organ or tissue models, animal tests) are followed by in silico/in vitro analysis for target and mechanism identification. On the contrary, the more recent reverse pharmacology approach consists of an in silico/in vitro screening of a large libraries of compounds and the "hits" are selected for further in vivo characterization [53].

In this review we will focus on an important class of secondary metabolites derived from plants, i.e., terpenes, that are under investigation for their antimicrobial activity, especially against antibiotic-resistant strains. The data we report in the next paragraphs were obtained from research groups all over the world and summarize the in vitro antimicrobial activity of these compounds, highlighting their high potentiality as new antimicrobials. This class of PDNPs has already been studied and commercialized for other important human diseases as proved by the high number of clinical trials (Table 1) and marketed drugs (Table 2). 
Table 1. Plant-derived terpenes in clinical trials (CT). Includes Not yet recruiting, Recruiting, Enrolling by invitation, Active not recruiting, Suspended and Completed trials of these compounds, parent extracts, or derivatives, applied alone or as a mixture with other constituents. Determined from www.clinicaltrials.gov on 14 May 2020.

\begin{tabular}{|c|c|c|c|c|}
\hline Compound & Plant Source & Number of CT & Medicinal Purposes & Ref. \\
\hline Andrographolide & Andrographis paniculata & 7 & $\begin{array}{l}\text { Squamous Cell Carcinoma of Esophagus, Multiple Sclerosis, Cognitive Impairment, } \\
\text { Migraine Disorders, Arthritis Rheumatoid, Acute Respiratory Infections. } \\
\text { Schizophrenia, Malaria, Plasmodium Falciparum Malaria, Malaria in Pregnancy, } \\
\text { Uncomplicated Falciparum Malaria, Vivax Malaria, Human Papilloma Virus }\end{array}$ & [79] \\
\hline Artemisinin & Artemisia annua $\mathrm{L}$. & 161 & $\begin{array}{l}\text { Infection, Pre-Cancerous Dysplasia, Cervical Dysplasia, Metastatic Breast Cancer, } \\
\text { Cytomegalovirus Infections, Increased Drug Resistance, Colorectal Cancer, Bowel }\end{array}$ & {$[64,80,81]$} \\
\hline Betulin & Betula spp. & 4 & Epidermolysis Bullosa, Wounds, Burns. & [82] \\
\hline Betulinic acid & Betula pubescens, Hypericum lanceolatum & 2 & Dysplastic Nevus Syndrome, Anxiety, Psychological Stress. & {$[82,83]$} \\
\hline Boswellic acid & Boswellia serrata & 4 & $\begin{array}{c}\text { Relapsing Remitting Multiple Sclerosis, Renal Stones, Knee Osteoarthritis, Joint } \\
\text { Pain, Stiffness. }\end{array}$ & [79] \\
\hline Geraniol & $\begin{array}{l}\text { Cinnamomum } \\
\text { tenuipilum, Valeriana officinalis }\end{array}$ & 2 & Coronary Artery Disease, Uterine Cervical Dysplasia, Papillomavirus Infections. & [84] \\
\hline Ginkgolides & Ginkgo biloba & 7 & $\begin{array}{l}\text { Intravenous Alteplase Thrombolysis, Neurological Improving, Allergy, } \\
\text { Ischemic Stroke. }\end{array}$ & [85] \\
\hline Gossypol & Gossypium hirsutum L. & 21 & $\begin{array}{c}\text { Recurrent Adrenocortical Carcinoma, Stage III and IV Adrenocortical Carcinoma, } \\
\text { Extensive Stage Small Cell Lung Cancer, Unspecified Adult Solid Tumor, Adult } \\
\text { Glioblastoma, Adult Gliosarcoma, Recurrent Adult Brain Tumor, Chronic } \\
\text { Lymphocytic Leukemia, Recurrent Chronic Lymphocytic Leukemia, Follicular } \\
\text { Lymphoma, Adenocarcinoma of the Prostate, Prostate Cancer, Diffuse Large Cell } \\
\text { Lymphoma, Mantle Cell Lymphoma, Laryngeal Cancer, Brain and Central Nervous } \\
\text { System Tumors. }\end{array}$ & [80] \\
\hline Limonene & $\begin{array}{l}\text { Citrus spp., } \\
\text { Apium graveolens }\end{array}$ & 10 & Breast Cancer, Allergic Contact Dermatitis Due to Cosmetics, Obesity. & [86] \\
\hline Lupeol & Hymenocardia acida & 2 & $\begin{array}{l}\text { Acne. } \\
\text { HIV-infection/AIDS, Advanced Cancer, Gastric Cancer, Breast Cancer, Pancreatic }\end{array}$ & [83] \\
\hline Triptolide & Tripterygium wilfordii & 4 & $\begin{array}{c}\text { Cancer, Prostate Cancer, Metastatic Colorectal Cancer, Solid Tumor, Solid } \\
\text { Carcinoma, Solid Carcinoma of Stomach, Cancer of Stomach. }\end{array}$ & {$[64,85,87]$} \\
\hline Ursolic acid & $\begin{array}{l}\text { Rosmarinus officinalis, Malus domestica, } \\
\text { Salvia officinalis, Thymus vulgaris }\end{array}$ & 2 & Metabolic Syndrome X, Sarcopenia. & [88] \\
\hline
\end{tabular}


Table 2. Plant-derived terpenes approved for therapeutic use. Additional resources from https://www.drugs.com/ and https://www.drugbank.ca/

\begin{tabular}{cccc}
\hline Compound & Trade Name & Plant Source & Medicinal Purposes \\
\hline Arglabin & Arglabin & Artemisia glabella & Cancer chemotherapy. \\
Artemisinin & Artemisinin & Artemisia annua $\mathrm{L}$. & Antiprotozoal agent: Antimalarial. \\
Docetaxel & Taxotere & Taxus baccata & Treatment of head, neck, stomach, lung, prostate, \\
breast and ovarian cancers. & [80,81] & Actinic keratosis. \\
Ingenol mebutate & Picato & Euphorbia peplus $\mathrm{L}$. & Chemotherapeutic agent for many types of cancer. \\
Paclitaxel & Taxol $^{\circledR}$, Paxene ${ }^{\circledR}$, Abraxane, Nanoxel & Taxus brevifolia Nutt. & {$[80,87]$} \\
\hline
\end{tabular}




\section{Terpenes}

Terpenes are the most numerous and structurally diverse class of natural products. These compounds are characterized by different carbon skeletons, but, despite structural differences, all terpenes are unified by a common biosynthetic pathway: the fusion of five-carbon isoprene units, the basic structural unit of terpenes. Since the last century, the biosynthetic process of terpenes was explained by the isoprene rule, which states that all terpenes derive from the ordered head-to-tail joining of isoprene units. A head-to-tail fusion is the most common; however, non-head-to-tail condensation of isoprene units also occurs [90,91]. It is useful to divide terpenes into classes according to the number of isoprene units they are biogenetically made of. They are classified as hemiterpenes (one isoprenoid unit); monoterpenes (two isoprenoid unites); sesquiterpenes (three isoprenoid unites); diterpenes (four isoprenoid unites); sesteterpenes (five isoprenoid unites); triterpenes (six isoprenoid unites) and tetraterpenes (eight isoprenoid unites) [92]. As reported in the literature, several terpenes have been employed as important pharmaceutical agents with anti-inflammatory [93], anti-viral [94], anti-diabetic [95], anti-tumor [96] and antibacterial activities [97]. Notably, terpenes have played a pivotal role in the fight against antibiotic resistance showing promising antibacterial potential against multi-resistant strains or acting as potentiators for antimicrobials by exhibiting synergistic effects [98-100]. Due to their low yield from natural source, semi-synthetic and synthetic derivatives with improved biological properties have been developed [101,102]. The first step in studying medicinal plants is the preparation of plant samples to preserve the secondary metabolites prior to extraction. Compounds from different parts of a plant such as leaves, barks, roots, fruits and flowers can be extracted from fresh or dried plants material. Traditional methods such as maceration and Soxhlet extraction are widely employed in the studies reported in this review. First, the leaves are properly treated, dried and separated from foreign materials including soil, pebbles and other matters unsuitable for the solid-liquid extraction process. The fresh material is then air-dried, macerated usually by using a suitable solvent that, due to its polarity, is able to extract polar as well as less polar organic compounds. The obtained solution is the desired extract. In each case, extensive purifications of the obtained extract are performed by preparative Thin Layer Chromatography (TLC) or classic column chromatography or by advanced technology such as flash chromatography and High Performance Liquid Chromatography (HPLC) [103-126].

The structures and the purity of all terpenes reported in this review were unambiguously confirmed through nuclear magnetic resonance (NMR) spectroscopy, and by electrospray ionization mass spectrometry (MS) [103-128]. Most of the terpenes reported are known and their multiple chiral centers were assigned according to the literature. When chirality is not reported, terpenes were tested as racemic form (see Table 3) [103,107,126,129]. In some studies, the authors investigated the extract chemical composition by Gas Chromatography-mass spectrometry (GC/MS) [129]. The chemical structure of the most representative terpenes examined in this review is shown in Table 3. 
Table 3. Summary of triterpenes with antimicrobial activity against resistant strains.

\begin{tabular}{|c|c|c|c|c|c|c|}
\hline N. & Common Name & $\begin{array}{l}\text { Chemical } \\
\text { Structure }\end{array}$ & $\begin{array}{c}\text { Tested } \\
\text { Microorganism }\end{array}$ & $\begin{array}{c}\text { Antimicrobial } \\
\text { Effect }\end{array}$ & Source & Ref. \\
\hline \multicolumn{7}{|c|}{ Monoterpenes } \\
\hline 1 & Geraniol & & E. aerogenes & Efflux Pump Inhibitor & $\begin{array}{c}\text { Species: } \\
\text { Helichrysum italicum }\end{array}$ & [130] \\
\hline 2 & Camphor & & $\begin{array}{c}\text { S. aureus } \\
\text { E. coli } \\
\text { E. aerogenes } \\
\text { P. aeruginosa } \\
\text { K. pneumoniae } \\
\text { K. oxytoca } \\
\text { Salmonella spp. } \\
\text { A. baumannii } \\
\text { E. cloacae }\end{array}$ & Growth inhibition & $\begin{array}{c}\text { Species: } \\
\text { Lavandula pedunculata subsp.atlantica } \\
\text { (BRAUN-BLANQ) }\end{array}$ & [129] \\
\hline 3 & Linalool & & $\begin{array}{c}\text { S. aureus } \\
\text { E. coli } \\
\text { P. aeruginosa } \\
\text { K. pneumoniae } \\
\text { K. oxytoca } \\
\text { Salmonella spp. } \\
\text { A. baumannii } \\
\text { E. cloacae }\end{array}$ & Growth inhibition & $\begin{array}{c}\text { Species: } \\
\text { Lavandula pedunculata subsp.atlantica } \\
\text { (BRAUN-BLANQ) }\end{array}$ & [129] \\
\hline 4 & Terpinen-4-ol & & $\begin{array}{c}\text { S. aureus } \\
\text { E. coli } \\
\text { P. aeruginosa } \\
\text { K. pneumoniae } \\
\text { K. oxytoca } \\
\text { Salmonella spp } \\
\text { A. baumannii } \\
\text { E. cloacae }\end{array}$ & Growth inhibition & $\begin{array}{c}\text { Species: } \\
\text { Lavandula pedunculata subsp.atlantica } \\
\text { (BRAUN-BLANQ) }\end{array}$ & [129] \\
\hline
\end{tabular}


Table 3. Cont.

\begin{tabular}{|c|c|c|c|c|c|c|}
\hline N. & Common Name & $\begin{array}{l}\text { Chemical } \\
\text { Structure }\end{array}$ & $\begin{array}{c}\text { Tested } \\
\text { Microorganism }\end{array}$ & $\begin{array}{c}\text { Antimicrobial } \\
\text { Effect }\end{array}$ & Source & Ref. \\
\hline 5 & Borneol & & $\begin{array}{c}\text { S. aureus } \\
\text { E. coli } \\
\text { E. aerogenes } \\
\text { P. aeruginosa } \\
\text { K. pneumoniae } \\
\text { K. oxytoca } \\
\text { A. baumannii } \\
\text { E. cloacae }\end{array}$ & Growth inhibition & $\begin{array}{c}\text { Species: } \\
\text { Lavandula pedunculata subsp.atlantica } \\
\text { (BRAUN-BLANQ) }\end{array}$ & [129] \\
\hline 6 & Fenchone & & $\begin{array}{l}\text { S. aureus } \\
\text { E. coli } \\
\text { E. aerogenes } \\
\text { P. aeruginosa } \\
\text { K. pneumoniae } \\
\text { K. oxytoca } \\
\text { Salmonella spp. } \\
\text { A. baumannii } \\
\text { E. cloacae }\end{array}$ & Growth inhibition & $\begin{array}{c}\text { Species: } \\
\text { Lavandula pedunculata subsp.atlantica } \\
\text { (BRAUN-BLANQ) }\end{array}$ & [129] \\
\hline \multicolumn{7}{|c|}{ Sesquiterpenes } \\
\hline 7 & Farnesyl amine 2 & & S.aureus & Growth inhibition & & [127] \\
\hline 8 & $\begin{array}{c}\text { Farnesyl } \\
\text { phosphoramidothioic } \\
\text { acid } 6\end{array}$ & & S. aureus & Growth inhibition & & [127] \\
\hline 9 & Arnicolide D & & $\begin{array}{l}\text { MRSA } \\
\text { MSSA }\end{array}$ & Growth inhibition & $\begin{array}{c}\text { Species: } \\
\text { Centipeda minima }\end{array}$ & [103] \\
\hline
\end{tabular}


Table 3. Cont.

\begin{tabular}{|c|c|c|c|c|c|c|}
\hline N. & Common Name & $\begin{array}{l}\text { Chemical } \\
\text { Structure }\end{array}$ & $\begin{array}{c}\text { Tested } \\
\text { Microorganism }\end{array}$ & $\begin{array}{c}\text { Antimicrobial } \\
\text { Effect }\end{array}$ & Source & Ref. \\
\hline 10 & Arnicolide C & & $\begin{array}{l}\text { MRSA } \\
\text { MSSA }\end{array}$ & Growth inhibition & $\begin{array}{c}\text { Species: } \\
\text { Centipeda minima }\end{array}$ & [103] \\
\hline 11 & Guaianolide 5 & & MRSA & Growth inhibition & $\begin{array}{c}\text { Species: } \\
\text { Artemisia gilvescens }\end{array}$ & [104] \\
\hline 12 & Dehydroleucodine & & MRSA & Growth inhibition & $\begin{array}{c}\text { Species: } \\
\text { Gynoxys verrucosa }\end{array}$ & [105] \\
\hline 13 & Xanthorrhizol & & $\begin{array}{l}\text { MRSA } \\
\text { E. coli }\end{array}$ & Growth inhibition & $\begin{array}{c}\text { Species: } \\
\text { Cinnamomum iners }\end{array}$ & [106] \\
\hline \multicolumn{7}{|c|}{ Diterpenes } \\
\hline 14 & Vitexolide A & & S. aureus & Growth inhibition & $\begin{array}{c}\text { Species: } \\
\text { Vitex vestita }\end{array}$ & [107] \\
\hline
\end{tabular}


Table 3. Cont

\begin{tabular}{|c|c|c|c|c|c|c|}
\hline N. & Common Name & $\begin{array}{l}\text { Chemical } \\
\text { Structure }\end{array}$ & $\begin{array}{c}\text { Tested } \\
\text { Microorganism }\end{array}$ & $\begin{array}{c}\text { Antimicrobial } \\
\text { Effect }\end{array}$ & Source & Ref. \\
\hline 15 & Acuminolide & & S. aureus & Growth inhibition & $\begin{array}{l}\text { Species: } \\
\text { Vitex vestita }\end{array}$ & [107] \\
\hline 16 & 12-epivitexolide A & & S. aureus & Growth inhibition & $\begin{array}{l}\text { Species: } \\
\text { Vitex vestita }\end{array}$ & [107] \\
\hline 17 & 8(17),12E,14-labdatrien-6,19-olide & & MRSA & Growth inhibition & $\begin{array}{l}\text { Species: } \\
\text { Salvia leriifolia }\end{array}$ & [108] \\
\hline 18 & $\begin{array}{c}8(17), 11(\mathrm{Z}), 13(E)- \\
\text { trien-15,19-dioic acid }\end{array}$ & & MRSA & Growth inhibition & $\begin{array}{c}\text { Species: } \\
\text { Caesalpinia decapetala }\end{array}$ & [109] \\
\hline
\end{tabular}


Table 3. Cont

\begin{tabular}{|c|c|c|c|c|c|c|}
\hline N. & Common Name & $\begin{array}{l}\text { Chemical } \\
\text { Structure }\end{array}$ & $\begin{array}{c}\text { Tested } \\
\text { Microorganism }\end{array}$ & $\begin{array}{c}\text { Antimicrobial } \\
\text { Effect }\end{array}$ & Source & Ref. \\
\hline 19 & (E)-8(17),12-labdadiene-15,16-dial & & $\begin{array}{l}\text { S. aureus } \\
\text { MRSA }\end{array}$ & Growth inhibition & $\begin{array}{c}\text { Species: } \\
\text { Zingiber montanum }\end{array}$ & [110] \\
\hline 20 & $\begin{array}{c}\text { 16 } \alpha \text {-hydroxycleroda-3, } 13 \\
\text { (14)-Z-dien-15, 16-olide (CD) }\end{array}$ & & MRSA & Growth inhibition & $\begin{array}{c}\text { Species: } \\
\text { Polyathia longifolia }\end{array}$ & [128] \\
\hline 21 & Coleon U & & $\begin{array}{l}\text { MRSA } \\
\text { VRE }\end{array}$ & Growth inhibition & $\begin{array}{c}\text { Species: } \\
\text { Plectranthus grandidentatus Plectranthus } \\
\text { hereroensis }\end{array}$ & [111] \\
\hline 22 & 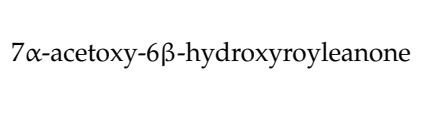 & & $\begin{array}{l}\text { MRSA } \\
\text { VRE }\end{array}$ & Growth inhibition & $\begin{array}{c}\text { Species: } \\
\text { Plectranthus grandidentatus Plectranthus } \\
\text { hereroensis }\end{array}$ & [111] \\
\hline
\end{tabular}


Table 3. Cont

\begin{tabular}{|c|c|c|c|c|c|c|}
\hline N. & Common Name & $\begin{array}{l}\text { Chemical } \\
\text { Structure }\end{array}$ & $\begin{array}{c}\text { Tested } \\
\text { Microorganism }\end{array}$ & $\begin{array}{l}\text { Antimicrobial } \\
\text { Effect }\end{array}$ & Source & Ref. \\
\hline 23 & Horminone & & $\begin{array}{l}\text { MRSA } \\
\text { VRE }\end{array}$ & Growth inhibition & $\begin{array}{c}\text { Species: } \\
\text { Plectranthus grandidentatus Plectranthus } \\
\text { hereroensis }\end{array}$ & [111] \\
\hline 24 & $\begin{array}{c}7 \alpha, 12 \text {-dihydroxy-17(15-16)abeo-abieta- } 8 \\
\text { 12,16-triene-11,14-dione }\end{array}$ & & $\begin{array}{l}\text { MRSA } \\
\text { VRE }\end{array}$ & Growth inhibition & $\begin{array}{c}\text { Species: } \\
\text { Plectranthus grandidentatus Plectranthus } \\
\text { hereroensis }\end{array}$ & [111] \\
\hline 25 & $\begin{array}{l}\text { 16-Acetoxy-7 } \alpha, 12 \text {-dihydroxy- } 8 \text {, } \\
\text { 12-abietadiene-11,14-dione }\end{array}$ & & $\begin{array}{l}\text { MRSA } \\
\text { VRE }\end{array}$ & Growth inhibition & $\begin{array}{c}\text { Species: } \\
\text { Plectranthus grandidentatus Plectranthus } \\
\text { hereroensis }\end{array}$ & [111] \\
\hline 26 & Sincoetsin C & & MRSA & Growth inhibition & $\begin{array}{c}\text { Species: } \\
\text { Coleus blumei Benth. }\end{array}$ & [112] \\
\hline
\end{tabular}


Table 3. Cont

\begin{tabular}{|c|c|c|c|c|c|c|}
\hline N. & Common Name & $\begin{array}{l}\text { Chemical } \\
\text { Structure }\end{array}$ & $\begin{array}{c}\text { Tested } \\
\text { Microorganism }\end{array}$ & $\begin{array}{l}\text { Antimicrobial } \\
\text { Effect }\end{array}$ & Source & Ref. \\
\hline 27 & ent-8 (14),15-pimaradien-3 $\beta$-ol & & $\begin{array}{l}\text { S. aureus } \\
\text { S. capitis } \\
\text { S. haemolyticus } \\
\text { E. faecalis } \\
\text { E. epidermidis } \\
\text { S. pneumoniae }\end{array}$ & Cell membrane disruption & $\begin{array}{c}\text { Species: } \\
\text { Viguiera arenaria }\end{array}$ & [113] \\
\hline 28 & rel-15(C),16-epoxy-7R-hydroxypimar-8,14ene & & MRSA & Growth inhibition & $\begin{array}{c}\text { Species: } \\
\text { Plectranthus ernstii }\end{array}$ & [114] \\
\hline 29 & rel-15(C),16-epoxy-7-oxopimar-8,14-ene & & MRSA & Growth inhibition & $\begin{array}{c}\text { Species: } \\
\text { Plectranthus ernstii }\end{array}$ & [114] \\
\hline 30 & 1R,11S-dihydroxy-8R,13R-epoxylabd-14-ene & & MRSA & Growth inhibition & $\begin{array}{c}\text { Species: } \\
\text { Plectranthus ernstii }\end{array}$ & [114] \\
\hline 31 & Isopimaric acid & & MRSA & Growth inhibition & $\begin{array}{l}\text { Species: } \\
\text { Pinus nigra }\end{array}$ & [115] \\
\hline
\end{tabular}


Table 3. Cont

\begin{tabular}{|c|c|c|c|c|c|c|}
\hline N. & Common Name & $\begin{array}{l}\text { Chemical } \\
\text { Structure }\end{array}$ & $\begin{array}{c}\text { Tested } \\
\text { Microorganism }\end{array}$ & $\begin{array}{c}\text { Antimicrobial } \\
\text { Effect }\end{array}$ & Source & Ref. \\
\hline 32 & ent-kaurenoic acid & & $\begin{array}{l}\text { S. aureus } \\
\text { S. capitis } \\
\text { S. epidermidis } \\
\text { S. haemolyticus } \\
\text { E. faecalis } \\
\text { MRSA }\end{array}$ & Cell membrane disruption & $\begin{array}{c}\text { Species: } \\
\text { Mikania glomerate } \\
\text { Citrus reticulate }\end{array}$ & $\begin{array}{l}{[116,} \\
117]\end{array}$ \\
\hline 33 & ent-pimaradienoic acid & & $\begin{array}{c}\text { S. aureus } \\
\text { S. capitis } \\
\text { S. epidermidis } \\
\text { S. haemolyticus } \\
\text { E. faecalis }\end{array}$ & Cell membrane disruption & $\begin{array}{c}\text { Species: } \\
\text { Viguiera arenaria }\end{array}$ & [116] \\
\hline 34 & semisynthetic derivative of 33 & & $\begin{array}{c}\text { S. aureus } \\
\text { S. capitis } \\
\text { S. epidermidis }\end{array}$ & Cell membrane disruption & & [116] \\
\hline 35 & ent-beyer-15-en-18-O-oxalate (BBN149) & & $\begin{array}{l}\text { P. aeruginosa } \\
\text { K. pneumoniae }\end{array}$ & Growth inhibition & $\begin{array}{l}\text { Species: } \\
\text { Fabiana densa var ramulosa }\end{array}$ & [118] \\
\hline
\end{tabular}


Table 3. Cont

\begin{tabular}{|c|c|c|c|c|c|c|}
\hline N. & Common Name & $\begin{array}{l}\text { Chemical } \\
\text { Structure }\end{array}$ & $\begin{array}{c}\text { Tested } \\
\text { Microorganism }\end{array}$ & $\begin{array}{c}\text { Antimicrobial } \\
\text { Effect }\end{array}$ & Source & Ref. \\
\hline \multicolumn{7}{|c|}{ Triterpenes } \\
\hline 36 & $\alpha$ - Amyrin & & $\begin{array}{l}\text { MSSA } \\
\text { MRSA }\end{array}$ & Growth inhibition & & [119] \\
\hline 37 & Betulinic acid & & $\begin{array}{l}\text { MSSA } \\
\text { MRSA }\end{array}$ & Growth inhibition & & [119] \\
\hline 38 & Betulinaldehyde & & $\begin{array}{l}\text { MSSA } \\
\text { MRSA }\end{array}$ & Growth inhibition & & [119] \\
\hline 39 & Oleanolic acid & & $\begin{array}{l}\text { MRSA } \\
\text { VRE }\end{array}$ & Growth inhibition & $\begin{array}{c}\text { Species: } \\
\text { Salvia officinalis (Sage) }\end{array}$ & [120] \\
\hline
\end{tabular}


Table 3. Cont

\begin{tabular}{|c|c|c|c|c|c|c|}
\hline N. & Common Name & $\begin{array}{l}\text { Chemical } \\
\text { Structure }\end{array}$ & $\begin{array}{c}\text { Tested } \\
\text { Microorganism }\end{array}$ & $\begin{array}{c}\text { Antimicrobial } \\
\text { Effect }\end{array}$ & Source & Ref. \\
\hline 40 & Ursolic acid & & $\begin{array}{l}\text { MRSA } \\
\text { VRE } \\
\text { NASEC } \\
\text { NAREC }\end{array}$ & Growth inhibition & $\begin{array}{c}\text { Species: } \\
\text { Salvia officinalis (Sage) } \\
\text { Eucalyptus tereticornis } \\
\text { Alstonia scholaris }\end{array}$ & [120-122] \\
\hline 41 & Butyl ester of ursolic acid & & $\begin{array}{l}\text { NASEC } \\
\text { NAREC }\end{array}$ & Growth inhibition & & [121] \\
\hline 42 & Isopropyl ester of ursolic acid & & $\begin{array}{l}\text { NASEC } \\
\text { NAREC }\end{array}$ & Growth inhibition & & [121] \\
\hline 43 & $18 \beta$-glycyrrhetinic acid (18ß-GA) & & MRSA & Growth inhibition & $\begin{array}{c}\text { Species: } \\
\text { Glycyrrhiza glabra }\end{array}$ & [131] \\
\hline
\end{tabular}


Table 3. Cont.

\begin{tabular}{|c|c|c|c|c|c|c|}
\hline N. & Common Name & $\begin{array}{l}\text { Chemical } \\
\text { Structure }\end{array}$ & $\begin{array}{c}\text { Tested } \\
\text { Microorganism }\end{array}$ & $\begin{array}{l}\text { Antimicrobial } \\
\text { Effect }\end{array}$ & Source & Ref. \\
\hline 44 & $\begin{array}{l}3 \beta, 6 \beta, 16 \beta \text {-trihydroxylup-20(29)-ene } \\
\text { (CLF1) }\end{array}$ & & $\begin{array}{l}\text { S. aureus } \\
\text { E. coli }\end{array}$ & Growth inhibition & $\begin{array}{l}\text { Species: } \\
\text { Combretum leprosum }\end{array}$ & [123] \\
\hline 45 & 16R-hydroxymollic & & $\begin{array}{l}\text { VRE } \\
\text { MRSA }\end{array}$ & Growth inhibition & $\begin{array}{c}\text { Species: } \\
\text { Acalypha communis }\end{array}$ & [124] \\
\hline 46 & 15R-hydroxymollic & & VRE & Growth inhibition & $\begin{array}{l}\text { Species: } \\
\text { Acalypha communis }\end{array}$ & [124] \\
\hline 47 & $\begin{array}{c}7 \beta, 16 \beta \text {-dihydroxy-1,23-dideoxyjessic } \\
\text { acids }\end{array}$ & & VRE & Growth inhibition & $\begin{array}{c}\text { Species: } \\
\text { Acalypha communis }\end{array}$ & [124] \\
\hline
\end{tabular}


Table 3. Cont.

\begin{tabular}{|c|c|c|c|c|c|c|}
\hline N. & Common Name & $\begin{array}{l}\text { Chemical } \\
\text { Structure }\end{array}$ & $\begin{array}{c}\text { Tested } \\
\text { Microorganism }\end{array}$ & $\begin{array}{c}\text { Antimicrobial } \\
\text { Effect }\end{array}$ & Source & Ref. \\
\hline 48 & Aphagrandinoid D & & MRSA & Growth inhibition & $\begin{array}{c}\text { Species: } \\
\text { Acacia grandifolia }\end{array}$ & [125] \\
\hline 49 & $3 \beta$-hydroxylanosta-9,24-dien-21-oic acid & & $\begin{array}{l}\text { S. aureus } \\
\text { P. mirabilis } \\
\text { Salmonella }\end{array}$ & Growth inhibition & $\begin{array}{c}\text { Species: } \\
\text { Protorhus longifolia }\end{array}$ & [126] \\
\hline 50 & methyl-3 $\beta$-hydroxylanosta-9,24-dien-21-oate & & $\begin{array}{l}\text { S. aureus } \\
\text { P. mirabilis } \\
\text { Salmonella }\end{array}$ & Growth inhibition & $\begin{array}{c}\text { Species: } \\
\text { Protorhus longifolia }\end{array}$ & [126] \\
\hline
\end{tabular}

MRSA, methicillin-resistant S. aureus; MSSA, methicillin sensitive S. aureus; VRE, vancomycin-resistant Enterococcus faecalis; NASEC, nalidixic acid-sensitive E. coli; NAREC, nalidixic acid-resistant E. coli. 


\subsection{Monoterpenes}

Monoterpenes are the main constituents of essential oils and they are responsible of the flavor and aroma of plant from which they are extracted. They are formed by the dimerization of isoprene units and, based on the arrangement of their carbon skeleton, they are grouped into acyclic and cyclic structures [132]. It is also useful to classify monoterpenes in line with their different chemical functionalities, including alcohol (such as linalool and geraniol), aldehyde (such as citral and citronellal), phenol (such as thymol and carvacrol), ketone (such as carvone and camphor), ether (such as eucalyptol) and hydrocarbon (such as cymene, pinene, limonene, and phellandrene) groups [92]. It has been long recognized that monoterpenes possess antimicrobial activity. Griffin et al. investigated the relationships between the structure/molecular properties and the antimicrobial activity of terpenes. They found that hydrogen bonding parameters are associated with their biological activity, in all cases [133]. Several oxygenate monoterpenes such as carvacrol, thymol, menthol, and geraniol exerted antimicrobial activity against several Gram(+) and Gram(-) bacteria [134]. In particular, geraniol (Table 3, compound 1), an acyclic monoterpene featuring alcoholic functionalization, has been extensively studied for its promising antimicrobial activities against MDR strains. Lorenzi et al. reported the ability of the essential oil, obtained from Helichrysum italicum, to significantly reduce the resistance of three pathogenic MDR Gram(-) bacteria to chloramphenicol [130]. The authors demonstrated the ability of the $H$. italicum essential oil to reduce chloramphenicol resistance of an Enterobacter aerogenes strain EAEP289 that over-expresses its AcrAB efflux pump, supporting their hypothesis regarding a potential efflux pump inhibitor (EPI) as a mechanism of antimicrobial activity. Further investigations confirmed that the essential oil derived from H. italicum contains one or more chemical constituents with EPI activity. Accordingly, chloramphenicol susceptibility testing was performed in the presence of several components of the most active fraction from the essential oil and, among them, geraniol resulted to be the most potent compound in reducing chloramphenicol minimum growth inhibitory concentration (MIC) up to 16-fold for the EAEP289 strain [130]. In addition, geraniol completely reversed the chloramphenicol resistance in combination with the well-studied EPI, phenylalanine arginine $\beta$-naphthylamide (PA $\beta N)$ control. Interestingly, the efficiency of geraniol in reducing drug resistance was also observed towards $\beta$-lactams and the fluoroquinolone norfloxacin highlighting that geraniol modulates antibiotic resistance in Gram(-) bacteria by targeting efflux pumps mechanisms [130]. Recently, Sayout et al. evaluated the antibacterial activity and the chemical composition of the essential oils from two species of the genus Lavandula: L. tenuisecta Coss.exBall and Lavandula pedunculata subsp.atlantica (BRAUN-BLANQ) [129,135]. Essential oils from both species showed antibacterial activity against several microorganisms leading to an increased interest for Lavandula genus in the treatment of bacterial infections caused by MDR strains. The two species are rich of oxygenated monoterpenes and the major constituents are camphor, fenchone and 1,8-cineole. The authors investigated the relationship between terpenoid constituents and antibacterial activity in order to identify the compound(s) that are responsible for the antibacterial activity of Lavandula atlantica essential oil. Among them, one of the major constituents, camphor (Table 3, compound 2), a bicyclic monoterpene ketone, exhibited antimicrobial activity against all the tested strains. Several other oxygenated monoterpenes isolated from Lavandula atlantica essential oil showed a strong antimicrobial activity against most of the studied strains. Among them, linalool (Table 3, compound 3), an acyclic monoterpene alcohol, with MIC values ranging from $1.44 \mu \mathrm{g} / \mathrm{mL}$ to $3.83 \mu \mathrm{g} / \mathrm{mL}$; terpinen-4-ol (Table 3, compound 4), a cyclic monoterpene alcohol, with MIC values ranging from $0.78 \mu \mathrm{g} / \mathrm{mL}$ to 3.13-6.25 $\mu \mathrm{g} / \mathrm{mL}$; borneol (Table 3, compound 5), a bicyclic monoterpene alcohol, with MIC values ranging from $0.47 \mu \mathrm{g} / \mathrm{mL}$ to $3.75 \mu \mathrm{g} / \mathrm{mL}$; and fenchone (Table 3, compound 6), a bicyclic monoterpene ketone, with MIC values ranging from $1.06 \mu \mathrm{g} / \mathrm{mL}$ to $4.25 \mu \mathrm{g} / \mathrm{mL}[129,135]$.

\subsection{Sesquiterpenes}

Sesquiterpenes are composed of three isoprene units and they are widely studied for their biological activities. Farnesol is a compound of 15 carbon atoms and can be considered the precursor of acyclic 
sesquiterpenes. This sesquiterpene, has shown a significant antimicrobial activity against several bacteria, including Staphylococcus aureus and S. epidermidis, with the ability to inhibit biofilm formation of Streptococcus spp. [134]. Furthermore, it was found to potentiate the activity of $\beta$-lactam antibiotics against antibiotic-resistant bacteria [136]. Recently, Kim et al. reported that two synthetic derivatives of farnesol (Table 3, compounds 7 and 8) had an antimicrobial activity against three different methicillin-resistant S. aureus (MRSA) with MICs of $512 \mu \mathrm{g} / \mathrm{mL}$ and $256-512 \mu \mathrm{g} / \mathrm{mL}$ for compounds 8 and 7, respectively). In addition, they displayed an enhancer effect of $\beta$-lactam antibiotics. For instance, when used in combination with oxacillin against resistant strains, farnesol and their derivatives were able to reduce the oxacillin MIC of up to 128-fold. A lower, but still significant potentiating activity was also observed in combination with ampicillin [127]. Moreover, by cyclization reactions a wide variety of monocyclic, bicyclic and tricyclic compounds can be formed [132]. Lee et al. investigated the relationship between the sesquiterpene lactone structure and the antimicrobial activity. The authors demonstrated that the activity depends on the presence of a beta unsubstituted cyclopentenone ring moiety and how its saturation dramatically reduced activity [137]. Several compounds belonging to the largest class of sesquiterpene lactones, the pseudo-guaianolides were also identified [138]. Among them, Arnicolide D and Arnicolide C (Table 3, compounds 9 and 10), isolated from Centipeda minima, a medicinal plants of Nepal, showed activity against both MRSA and methicillin sensitive $S$. aureus (MSSA) with MIC values of $300 \mu \mathrm{g} / \mathrm{mL}$ versus MRSA, and $75 \mu \mathrm{g} / \mathrm{mL}$ and $38 \mu \mathrm{g} / \mathrm{mL}$ versus MSSA, respectively [103]. Furthermore, a related guaianolide 5 (Table 3, compound 11), isolated from Artemisia gilvescens manifested an excellent potential against a clinical strain of MRSA with a MIC value of $1.95 \mu \mathrm{g} / \mathrm{mL}$ [104]. Ordónez et al. studied the antimicrobial activity of two sesquiterpene lactones, belonging to the guainolide group, isolated from Gynoxys verrucosa, against six clinical isolates of $S$. aureus and S. epidermidis with different drug-resistance profiles. The sesquiterpene dehydroleucodine (Table 3, compound 12) exhibited antimicrobial activity against all staphylococcal isolates, including four methicillin-resistant strains with a minimum concentration inhibiting 50\% bacterial growth $\left(\mathrm{IC}_{50}\right)$ between 49 and $195 \mu \mathrm{g} / \mathrm{mL}$. Interestingly, the antimicrobial activity of dehydroleucodine and other structurally related sesquiterpene lactones, such as Arnicolide C, against MRSA and MSSA strains, suggested that the presence of a carbonyl moiety in the opposite side of the cycloheptane ring acts as a secondary hydrogen binding point and that the methylene group in the lactone ring significantly affects antimicrobial activity [105]. Among the phenolic sesquiterpenes, Xanthorrizol (Table 3, compound 13) from Cinnamomum iners, displayed significant antibacterial activity against MRSA with a MIC of $25 \mu \mathrm{g} / \mathrm{mL}$ [106]. Goncalves et al. inquired into the antibacterial effect of a wide range of antibiotics, such as tetracycline, erythromycin, penicillin, and vancomycin, when combined with nine sesquiterpenic compounds, e.g., hydrocarbons and alcohols, on two clinically relevant $S$. aureus and Escherichia coli strains with well-defined resistance-sensitive profiles. Several combinations of antibiotic-sesquiterpenic compounds increased the antibacterial activity of the antibiotics against S. aureus. For E. coli, an antagonistic effect was observed. Moreover, this study paved the way for the evaluation of sesquiterpenes as possible antibiotic enhancer against MRSA [139].

\subsection{Diterpenes}

Diterpenes consist of a chemically diverse group of compounds, all with a C20 carbon skeleton based on four isoprene units. They can be classified as linear, bicyclic, tricyclic, tetracyclic, pentacyclic or macrocyclic diterpenes depending on their skeletal core. A great number of diterpenes showed significant antimicrobial activity against MDR bacteria, and/or the ability to enhance the effectiveness of antibiotics when evaluated in combination with them against resistant strains [90,134,140]. Labdane-type bicyclic diterpenes, featuring a decalin system and a six-member ring which may be open or closed with an oxygen atom, proved an interesting antimicrobial activity against MDR strains [102]. Six new labdane-type diterpenes, isolated from the Malaysian species of Vitex vestita were tested for the first time against a panel of $46 \mathrm{Gram}(+)$ bacterial strains, both sensitive or resistant to conventional antibiotics. Interestingly, vitexolide A (Table 3, compound 14) resulted the most active compound with MICs ranging from 2 to $32 \mu \mathrm{g} / \mathrm{mL}$ against all tested microorganisms and 
a MIC of $4 \mu \mathrm{g} / \mathrm{mL}$ against the human MDR S. aureus strain CRBIP 21.21. Acumenolide (Table 3, compound 15) indicated an anti-Bacillus activity (MIC of $16 \mu \mathrm{g} / \mathrm{mL}$ ) while 12-epivitexolide A (Table 3, compound 16), the $\mathrm{C} 12$ epimer of vitexolide $\mathrm{A}$, and acuminolide, showed moderate antibacterial activity against a panel of $S$. aureus strains with MIC values of $16 \mu \mathrm{g} / \mathrm{mL}$. The structure-activity relationship analysis underlined that the presence of the $\gamma$-hydroxybutenolide moiety along with a C12 hydroxylation are essential for the antimicrobial activity [107]. Interestingly, another bicyclic diterpene with labdane-type scaffold, the 8(17),12E,14-labdatrien-6,19-olide (Table 3, compound 17), featuring a five-member lactone ring and three unsaturations, was isolated from Salvia leriifolia and showed a significant antimicrobial activity against a clinical MRSA strain with a MIC of $213 \mu \mathrm{M}$ [108]. A structurally related diterpene, 8(17),11(Z),13(E)-trien-15,19-dioic acid (Table 3, compound 18), isolated from Caesalpinia decapetala displayed a moderate antibacterial activity against a MRSA strain with a $\mathrm{IC}_{50}$ of $5.99 \mu \mathrm{g} / \mathrm{mL}$ [109]. Recently, Siddique et al. examined the antibacterial activity of a labdane diterpene, the (E)-8(17),12-labdadiene-15,16-dial (Table 3, compound 19) from Zingiber montanum, against a panel of clinical isolates of MDR S. aureus and MRSA and found out a potent activity with MICs ranging from 46 to $93 \mu \mathrm{g} / \mathrm{mL}$. In addition, the authors pointed out that the unsaturations (exomethylene C-8 and olefine C-12) and the two aldehyde groups at C-16 and C-17 on the diterpenoid's carbon skeleton could account for the significant activity against MRSA strains [110]. Gupta et al. reported the antimicrobial activity of a clerodane diterpene, $16 \alpha$-hydroxycleroda-3, 13 (14)-Z-dien-15, 16-olide (CD) (Table 3, compound 20), isolated from leaves of Polyathia longifolia, against seven clinical MRSA isolates with MICs in the range of $15.625-31.25 \mu \mathrm{g} / \mathrm{mL}(15.625 \mu \mathrm{g} / \mathrm{mL}$ against reference strain SA-96) [128]. The authors also investigated the synergistic interactions and the resistance-modifying potential of $\mathrm{CD}$ in combination with fluoroquinolones against these strains and its efflux pump inhibitory potential [141]. This diterpenoid enhanced the efficacy of norfloxacin, ciprofloxacin and ofloxacin against MRSA clinical isolates reducing up to 16-fold the MIC of norfloxacin against the MRSA clinical isolate ST2071. Furthermore, the results of time-kill kinetics revealed that $C D$ in combination with norfloxacin at their $\frac{1}{2} \mathrm{MIC}$ significantly reduced the viability of bacterial cells while flow cytometric analysis and spectrofluorometric assay clearly indicated that CD diterpene inhibited an MDR efflux pump [141]. The abietane-type is a class of tricyclic diterpenoid widespread in several botanical families. The aromatic abietanes are the largest group of naturally occurring abietanes. They are characterized by an aromatic $\mathrm{C}$ ring and a different degree of oxygenation at several positions. Generally, aromatic abietanes are not functionalized on the A-ring carbons. Most of them presents a different degree of oxidation in their B- and C-ring carbons [142]. Gaspar-Marques C. et al., investigated the antibacterial effect of several natural abietanes isolated from Plectranthus grandidentatus and P. hereroensis against MRSA and vancomycin-resistant Enterococcus faecalis (VRE). Diterpenes coleon U, $7 \alpha$-acetoxy-6 $\beta$-hydroxyroyleanone and horminone (Table 3, compound 21, 22 and 23) were the most active, with a remarkable activity against MRSA strains (MICs ranging from $0.98 \mu \mathrm{g} / \mathrm{mL}$ to $15.63 \mu \mathrm{g} / \mathrm{mL}$ ) and a more moderate activity against VRE strains (MIC values from 15.63 and $31.25 \mu \mathrm{g} / \mathrm{mL}$ ) [111]. Among the other abietanes tested, diterpenes 7 $\alpha, 12$-dihydroxy-17(15-16)abeo-abieta-8,12,16-triene-11,14-dione (Table 3, compound 24) and 16-acetoxy-7 $\alpha, 12$-dihydroxy-8,12-abietadiene-11,14-dione (Table 3, compound 25) showed a weaker activity, with MICs comprised between $15.63 \mu \mathrm{g} / \mathrm{mL}$ and $62.50 \mu \mathrm{g} / \mathrm{mL}$. The authors suggested that 12-hydroxy-p-benzoquinone moiety in ring $C$ together with an oxidized B ring at the C-6 and C-7 positions are essential for antimicrobial activity against MRSA and VRE. Furthermore, the most promising compound, coleon $\mathrm{U}$, consists of the more oxygenated and dehydrogenated chromophoric system through rings $\mathrm{B}$ and $\mathrm{C}$. Moreover, the presence of the $6 \beta$-hydroxyl and $7 \alpha$-acetoxy groups, as in $7 \alpha$-acetoxy- $6 \beta$-hydroxyroyleanone, or the oxidation at $\mathrm{C} 7$ position, as in horminone and abietanes 24 and 25, preserved a good antimicrobial activity [111]. Recently, Jurkaninovà S. and co-workers evaluated the anti-MRSA activity of abietane diterpenes from Coleus blumei Benth. Sincoetsin C (Table 3, compound 26) was the most potent compound with a MIC of $128 \mu \mathrm{g} / \mathrm{mL}$ against the MRSA strain CCM 4750 whereas the abietane glucosides did not show a valuable growth inhibition of MRSA, 
suggesting that the glycosylation of the hydroxyl group on the abietane skeleton suppresses the anti-MRSA activity [112]. Notably, Porto et al. studied the structure-antimicrobial activity relationships of pimarane-type diterpenes against MDRs, increasing the diversity of chemical structures by microbial transformation. Five tricyclic diterpene derivatives of the ent-8(14), 15-pimaradiene (PD), isolated from Viguiera arenaria, were obtained by fungal transformation in Aspergillus ochraceus. This allowed to introduce a reactive center into the poorly functionalized PD skeleton which was then tested against eight clinically isolated MDR bacteria. Interestingly, the diterpene derivative ent-8 (14), 15-pimaradien-3 $\beta$-ol (Table 3, compound 27) was much more effective than its biosynthetic precursor, with promising MIC values (lower than $10.0 \mu \mathrm{g} / \mathrm{mL}$ ) against a large variety of microbial pathogens. The time-kill curve experiments were carried out on MDR strain of S. aureus and showed that this compound was endowed with bactericidal action at all the evaluated concentrations (8.0, 16.0 and $24.0 \mu \mathrm{g} / \mathrm{mL}$ ) within $24 \mathrm{~h}$ of incubation. Additionally, it was tested in combination with vancomycin at the minimal bactericidal concentration. Time-kill curve profile of resulting combination denoted that the number of viable microorganisms was drastically reduced within the first six hours. Furthermore, by comparing structural features of the tested compounds with biological data, the authors established that the antimicrobial activity of pimarane-type diterpenes was ruled by structural factors other than the hydrogen-bond-donor (HDB) Urzúa hypothesis suggesting that the structural requirements responsible for an efficient antibacterial activity include a lipophilic structure, capable of insertion into the cell membrane and one hydrophilic group (HBD) that interacts with the phosphorylated headgroups of membrane phospholipids $[113,143]$. In 2009, Stavri and co-workers analyzed the antimicrobial activity of three new diterpenes, isolated from Plectranthus ernstii, against a panel of MRSA. Among them,

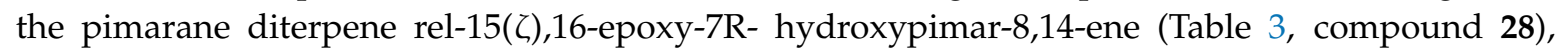
exhibited moderate antistaphylococcal activity against MDR strains with a MIC of $32 \mu \mathrm{g} / \mathrm{mL}$, whereas

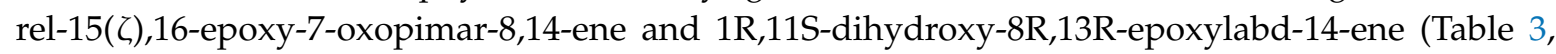
compound 29 and 30) showed a weaker activity. Surprisingly, a simple structural difference between the two pimarane diterpenes from 7-hydroxy to 7-oxo in the pimarane compound resulted in a loss of antistaphylococcal activity, presumably due to an increased lipophilicity and poorer uptake of the compound [114]. Among the pimarane-type diterpenes, Smith et al. reported the antimicrobial activity of isopimaric acid (Table 3, compound 31), from Pinus nigra, against strains with MDR efflux pumps and epidemic MRSA with MIC from $32 \mu \mathrm{g} / \mathrm{mL}$ to $64 \mu \mathrm{g} / \mathrm{mL}$ [115]. The authors also evaluated the activity of isopimaric acid in combination with tetracycline, norfloxacin and erythromycin or ofloxacin against the corresponding strains. However, no reduction of MIC was obtained for any of the antibiotics. Interestingly, isopimaric acid tested in combination with the efflux pump inhibitor reserpine $(20 \mu \mathrm{g} / \mathrm{mL})$ against two resistant strains, significantly increased the MIC for this compound. The authors postulated that isopimaric acid is not a substrate for these efflux pumps, but it is also possible that an antagonistic interaction with reserpine may render the antibiotics inactive. A molecular modeling study, performed by Zloh et al., reported the possible formation of complexes between MDR inhibitors and the substrates of efflux pumps. This would facilitate the entry of MDR inhibitors into the cell, following by complex dissociation, to allow the drug displaying its effect [144]. Based on this finding, it is possible that the complex formation is due to strong interactions, with a very slow rate of dissociation. This would lower the concentration of the free drug thus decreasing its anti-bacterial activity. Further investigations by Smith et al. supported this hypothesis highlighting a possible complex formation between diterpenes and reserpine responsible for a reduction in activity [115]. Recently, Soares A.C. and co-workers analyzed the antibacterial activity of two natural diterpenes against a set of seven clinically isolated MRD bacteria, including three S. aureus strains [116]. The two diterpenes, the tetracyclic ent-kaurenoic acid and the tricyclic ent-pimaradienoic acid (Table 3, compound 32 and 33), featuring a different basic skeleton, were isolated from Mikania glomerata and Viguiera arenaria, respectively, and selected for this study according to the Urzúa hypothesis. The biological assays gave MIC values lower than $13 \mu \mathrm{g} / \mathrm{mL}$, supporting the HBD Urzúa hypothesis. In order to perform structure-activity relationships studies the authors developed several semi-synthetic derivatives from both natural diterpenes. The hydrogenated 
product derivative of 33 (Table 3, compound 34), resulted to be even more efficient as an antimicrobial agent against MRSA and S. capitis with a MIC of $6.25 \mu \mathrm{g} / \mathrm{mL}$ [116]. Furthermore, Barbosa et al. reported the potent antibacterial activity of the ent-kaurenoic acid isolated from C. reticulata oleoresin against MRSA ( $\mathrm{IC}_{50} 3.4 \mu \mathrm{g} / \mathrm{mL}$ ) [117]. Interestingly, a docking-based virtual screening of an in-house library of natural products within the catalytic site of ArnT, the enzyme responsible for colistin resistance mediated by lipid A aminoarabinosylation in Pseudomonas aeruginosa, led to the identification of the ent-beyer-15-en-18-O-oxalate (BBN149, Table 3, compound 35), as promising inhibitor of ArnT [118]. This natural tetracyclic diterpene, featuring the ent-beyerene scaffold, was isolated from Fabiana densa var. ramulosa. The compound demonstrated to act as potent colistin adjuvant, potentiating its antibacterial activity up to 16-fold against colistin-resistant P. aeruginosa and K. pneumoniae isolates. The compound showed no activity against colistin-susceptible strains and no relevant toxicity towards human cells. The binding mode of BBN149 within the catalytic site of ArnT was evaluated by molecular docking simulations and identified two main binding poses. In both poses, the oxalyl group nicely overlaps with the crystallographic phosphate moiety and establishes H-bond interaction with the Lys85 residue, which was shown to be important for ArnT activity. Notably, this new insight made this diterpene a promising candidate for lead optimization of colistin resistance inhibitors with improved activity and/or pharmacological properties. Furthermore, natural analogs of the initial hit compound were isolated, and several semisynthetic analogs were designed and synthesized to afford structure-activity relationships (SAR). Currently, these data are covered by a patent [145].

\subsection{Triterpenes}

Triterpenes are composed by a carbon skeleton of six isoprene units and the main groups are represented by tetracyclic and pentacyclic structures. Pentacyclic triterpenes are all based on a 30-carbons skeleton comprising five six-membered rings (such as ursanes and lanostanes) or four six-membered rings and one five-membered ring (such as lupanes and hopanes). Interestingly, several pentacyclic triterpenes have been described for their antimicrobial activity [146,147]. Chung et al. reported the antimicrobial activity of $\alpha$-amyrin, betulinic acid and betulinaldehyde (Table 3 , compound 36, 37 and 38) against clinical isolates of MRSA and MSSA with MICs ranging from 64 to $512 \mu \mathrm{g} / \mathrm{mL}$ [119]. These pentacyclic triterpenes featured five six-membered rings, in the case of $\alpha$-amyrin, or four six-membered rings and one five-membered ring, for betulinic acid and betulinaldehyde. Noteworthy, although their MICs were higher than those of the commonly used antibiotics vancomycin and methicillin, the combinations of these drugs with such pentacyclic triterpenes reduced the MIC to a range of 0.05 to $50 \%$. Interestingly, the authors also demonstrated the potential synergistic activity of the standard antibiotics with these diterpenes on MRSA and MSSA [119]. Horiuchi et al. identify oleanolic acid, a pentacyclic triterpenoid related to betulinic acid, as the compound responsible for the antimicrobial activity of the Salvia officinalis (Sage) leaves extract against VRE. This pentacyclic triterpene (Table 3, compound 39), an olean-12-en-28-oic acid substituted by a beta-hydroxy group at position 3, displayed a good activity against VRE and MRSA strains with MIC values of $8 \mu \mathrm{g} / \mathrm{mL}$ and $16 \mu \mathrm{g} / \mathrm{mL}$, respectively. To get insight into structure-antimicrobial activity relationships, the authors tested structural related triterpenes and, among them, ursolic acid (Table 3, compound 40), the C19 epimer of oleanolic acid. It showed a higher antimicrobial activity against both VRE and MRSA strains with MIC values of $4 \mu \mathrm{g} / \mathrm{mL}$ and $8 \mu \mathrm{g} / \mathrm{mL}$, respectively [120]. Furthermore, ursolic acid, isolated from Eucalyptus tereticornis, did not exhibit antibacterial activity against the nalidixic acid-sensitive (NASEC) and nalidixic acid-resistant (NAREC) strains of E. coli, but in combination with nalidixic acid, this pentacyclic triterpene reduced the MIC of drug by two-fold (MIC of nalidixic acid alone was $6.25 \mu \mathrm{g} / \mathrm{mL}$ on NASEC and $100 \mu \mathrm{g} / \mathrm{mL}$ against NAREC). Based on these results, several semisynthetic derivatives of ursolic acid were developed and tested to evaluate their drug resistance reversal potential. Among them, the butyl and isopropyl ester of ursolic acid (Table 3, compound 41 and 42) reduced the MIC of nalidixic acid by 8-fold against NAREC and by 4- to 8-fold against NASEC [121]. Furthermore, in a study reported by Wang et al. on the antibacterial and synergistic activity of pentacyclic triterpenes isolated 
from Alstonia scholaris, ursolic acid showed a good antimicrobial activity against Gram $(+)$ pathogens including a MRSA strain, with a MIC value of $64 \mu \mathrm{g} / \mathrm{mL}$ and a synergistic effect with ampicillin and tetracycline against this strain [122]. A preliminary screening of a 350 compound proprietary in-house library containing marketed drugs and natural compounds to identify small molecules able to potentiate the antibacterial activity of some antibiotics against MRSA strains, led to the selection of $18 \beta$-glycyrrhetinic acid (18ß-GA, Table 3, compound 43), as the most active compound against S. aureus ATCC6538. It is a pentacyclic triterpene featuring five six-membered rings and found in nature together with its $18 \alpha$-epimer (18 $\alpha$-GA) as aglycons of glycyrrhizin in Glycyrrhiza glabra. The authors discovered a synergistic effect of $18 \beta-$ GA with several antibiotics against the MRSA strain LUH14616. Notably, this triterpene also potentiated the activity of tobramycin (16-32-fold), amikacin (8-fold) and polymyxin B (32-64-fold) against LUH21416, indicating $18 \beta-$ GA as a good antibiotic enhancer against MRSA [131]. Recently, the lupane triterpene 3 $3,6 \beta, 16 \beta$-trihydroxylup-20(29)-ene (CLF1, Table 3, compound 44), isolated from Combretum leprosum Mart. leaves, showed antibacterial action with clinical relevance against $10 \mathrm{MDR}$ strains with MIC $\geq 4 \mu \mathrm{g} / \mathrm{mL}$ and, more interestingly, synergistic effects were observed in combination with the antibiotic amikacin, reducing the MIC value from $100 \mu \mathrm{g} / \mathrm{mL}$ to $80 \mu \mathrm{g} / \mathrm{mL}$. In addition, this pentacyclic triterpene increased antimicrobial activity against MDR E. coli when associated with the antibiotics gentamicin and amikacin lowering MIC values from $20 \mu \mathrm{g} / \mathrm{mL}$ to $15 \mu \mathrm{g} / \mathrm{mL}$ and from $100 \mu \mathrm{g} / \mathrm{mL}$ to $60 \mu \mathrm{g} / \mathrm{mL}$ [123]. Noteworthy, the triterpenes with cycloartane skeleton were active against the antibiotic resistant clinical isolates [148]. These triterpenes have a unique pentacyclic structure characterized by cyclopropyl methylene group on the carbon skeleton. In a study reported by Gutierrez-Lugo et al., three cycloartane-type triterpenes i.e., 16R-hydroxymollic, 15R-hydroxymollic and 7 $\beta, 16 \beta$-dihydroxy-1,23-dideoxyjessic acids (Table 3, compounds 45, 46 and 47), isolated from the aerial parts of Acalypha communis, exhibited moderate antimicrobial activity against VRE with MIC values of $8 \mu \mathrm{g} / \mathrm{mL}, 32 \mu \mathrm{g} / \mathrm{mL}$ and $8 \mu \mathrm{g} / \mathrm{mL}$, respectively. In addition, 45 also displayed moderate activity against MRSA (MIC $64 \mu \mathrm{g} / \mathrm{mL}$ ) [124]. Furthermore, Wang et al. evaluated the antimicrobial activity of new five cycloartane-type triterpenes, isolated from Acacia grandifolia, against two MRSA strains. These compounds consisted in three new 29-nor-cycloart triterpenes aphagrandinoids A-C, and a new natural product, aphagrandinoid D (Table 3, compound 48), along with a structurally related known one (20R)-3 $\beta$-hydroxy-24,25,26,27-tetranor-5 $\alpha$-cycloartan-23,21-olid. Among these compounds, the last triterpene, belonging to the $3 \beta$-cycloartanol series, showed the strongest antibacterial activity against MRSA strains (MIC values of $1.57 \mu \mathrm{g} / \mathrm{mL}$ versus $25 \mathrm{or} 50 \mu \mathrm{g} / \mathrm{mL}$ ), despite a slightly weaker antimicrobial potency compared to the commercial drug vancomycin (MIC of $0.78 \mu \mathrm{g} / \mathrm{mL}$ ) [122]. Several triterpenes with tetracyclic structures were also found to be active against MDR strains. In line with this, Mosa et al. evaluated the antibacterial activity of two triterpenes i.e., 3ß-hydroxylanosta-9, 24-dien-21-oic acid and methyl-3ß-hydroxylanosta9,24-dienoate (Table 3, compound 49 and 50), isolated from Protorhus longifolia, against a panel of antibiotic resistant Gram(+) and $\operatorname{Gram}(-)$ bacteria. The triterpenes had an antibacterial activity comparable to that of conventional antibiotics against resistant strains of S. aureus [126].

\section{Conclusions}

Despite the discovery of antibiotics that has saved millions of lives from microbial infections, the latter continue to be a serious threat to public health due to the appearance of resistant strains. New molecules with antimicrobial activities are therefore urgently needed. Plant-derived terpenes represent an interesting class of molecules with a multitude of activities that make them of reference for drug-discovery as proven by the numerous clinical trials and drugs on the market ( Table 1; Table 2). Terpenes also possess antimicrobial activity and, in recent years, the studies we have reported have shown their ability to act also against antibiotic-resistant strains. This class of compounds therefore has all the potential for the development of new antimicrobials, especially in this post-antibiotic era in which we are living. 
Author Contributions: F.G. conceptualized the review. F.C., M.R.L., B.C. and D.Q. wrote the manuscript. C.D.P. and S.C. contributed in writing the manuscript and designed figures and tables. M.L.M. and B.B. critically revised the manuscript. All authors have red and agreed to the published version of the manuscript.

Funding: This work was supported from PON (Piano Operativo Nazionale) Grant ARS01_00432 PROGEMA, “Processi Green per l’Estrazione di Principi Attivi e la Depurazione di Matrici di Scarto e Non", 03/2018-09/2020 and PRIN 2017-“Targeting Hedgehog pathway: Virtual screening identification and sustainable synthesis of novel Smo and Gli inhibitors and their pharmacological drug delivery strategies for improved therapeutic effects in tumors" by the Italian Ministry of Education, University and Research (MIUR) and Sapienza University of Rome. This work was also supported by MIUR- Dipartimenti di Eccellenza- L. 232/2016 and Sapienza University of Rome (Project RM11816436113D8A).

Acknowledgments: The authors acknowledge networking contribution by the COST Action CM1407 "Challenging Organic Syntheses Inspired by Nature-From Natural Products Chemistry to Drug Discovery".

Conflicts of Interest: The authors declare no conflict of interest.

\section{References}

1. Robert, G. The discovery of penicillin-new insights after more than 75 years of clinical use. Emerg. Infect. Dis. J. 2017, 23, 849 .

2. Ribeiro da Cunha, B.; Fonseca, L.P.; Calado, C.R.C. Antibiotic discovery: Where have we come from, where do we go? Antibiotics 2019, 8, 45. [CrossRef] [PubMed]

3. Silver, L.L. Challenges of antibacterial discovery. Clin. Microbiol. Rev. 2011, 24, 71-109. [CrossRef] [PubMed]

4. Gould, K. Antibiotics: From prehistory to the present day. J Antimicrob. Chemother. 2016, 71, 572-575. [CrossRef]

5. Zappia, G.; Ingallina, C.; Ghirga, F.; Botta, B. Oxazolidin-2-ones: Antibacterial activity and chemistry. In Antimicrobials: New and Old Molecules in the Fight against Multi-Resistant Bacteria; Marinelli, F., Genilloud, O., Eds.; Springer: Berlin, Germany, 2014; pp. 247-266.

6. Rather, I.A.; Kim, B.C.; Bajpai, V.K.; Park, Y.H. Self-medication and antibiotic resistance: Crisis, current challenges, and prevention. Saudi. J. Biol. Sci. 2017, 24, 808-812. [CrossRef]

7. Dodds, D.R. Antibiotic resistance: A current epilogue. Biochem. Pharmacol. 2017, 134, 139-146. [CrossRef]

8. Katz, M.L.; Mueller, L.V.; Polyakov, M.; Weinstock, S.F. Where have all the antibiotic patents gone? Nat. Biotechnol. 2006, 24, 1529-1531. [CrossRef]

9. Kraus, C.N. Low hanging fruit in infectious disease drug development. Curr. Opin. Microbiol. 2008, 11, 434-438. [CrossRef]

10. Projan, S.J. Why is big pharma getting out of antibacterial drug discovery? Curr. Opin. Microbiol. 2003, 6, 427-430. [CrossRef]

11. Projan, S.J.; Shlaes, D.M. Antibacterial drug discovery: Is it all downhill from here? Clin. Microbiol. Infect. 2004, 10, 18-22. [CrossRef]

12. Spellberg, B.; Powers, J.H.; Brass, E.P.; Miller, L.G.; Edwards, J.E., Jr. Trends in antimicrobial drug development: Implications for the future. Clin. Infect. Dis. 2004, 38, 1279-1286. [CrossRef] [PubMed]

13. Cassini, A.; Hogberg, L.D.; Plachouras, D.; Quattrocchi, A.; Hoxha, A.; Simonsen, G.S.; Colomb-Cotinat, M.; Kretzschmar, M.E.; Devleesschauwer, B.; Cecchini, M.; et al. Attributable deaths and disability-adjusted life-years caused by infections with antibiotic-resistant bacteria in the eu and the european economic area in 2015: A population-level modelling analysis. Lancet Infect. Dis 2019, 19, 56-66. [CrossRef]

14. Renwick, M.J.; Brogan, D.M.; Mossialos, E. A systematic review and critical assessment of incentive strategies for discovery and development of novel antibiotics. J. Antibiot. (Tokyo) 2016, 69, 73-88. [CrossRef] [PubMed]

15. Tan, S.Y.; Tatsumura, Y. Alexander fleming (1881-1955): Discoverer of penicillin. Singap. Med. J. 2015, 56, 366-367. [CrossRef] [PubMed]

16. Reygaert, W.C. An overview of the antimicrobial resistance mechanisms of bacteria. AIMS Microbiol. 2018, 4, 482-501. [CrossRef] [PubMed]

17. Wood, T.K.; Knabel, S.J.; Kwan, B.W. Bacterial persister cell formation and dormancy. Appl. Environ. Microbiol. 2013, 79, 7116-7121. [CrossRef]

18. Keren, I.; Kaldalu, N.; Spoering, A.; Wang, Y.; Lewis, K. Persister cells and tolerance to antimicrobials. FEMS Microbiol. Lett. 2004, 230, 13-18. [CrossRef]

19. Martinez, J.L. General principles of antibiotic resistance in bacteria. Drug Discov. Today Technol. 2014, 11, 33-39. [CrossRef] 
20. Arzanlou, M.; Chai, W.C.; Venter, H. Intrinsic, adaptive and acquired antimicrobial resistance in gram-negative bacteria. Essays Biochem. 2017, 61, 49-59.

21. Cox, G.; Wright, G.D. Intrinsic antibiotic resistance: Mechanisms, origins, challenges and solutions. Int. J. Med. Microbiol. 2013, 303, 287-292. [CrossRef]

22. Blair, J.M.; Webber, M.A.; Baylay, A.J.; Ogbolu, D.O.; Piddock, L.J. Molecular mechanisms of antibiotic resistance. Nat. Rev. Microbiol. 2015, 13, 42-51. [CrossRef] [PubMed]

23. Coculescu, B.I. Antimicrobial resistance induced by genetic changes. J. Med. Life. 2009, 2, 114-123. [PubMed]

24. Davies, J.; Davies, D. Origins and evolution of antibiotic resistance. Microbiol. Mol. Biol. Rev. 2010, 74, 417-433. [CrossRef] [PubMed]

25. Maldonado, R.F.; Sa-Correia, I.; Valvano, M.A. Lipopolysaccharide modification in gram-negative bacteria during chronic infection. FEMS Microbiol. Rev. 2016, 40, 480-493. [CrossRef] [PubMed]

26. Blair, J.M.; Richmond, G.E.; Piddock, L.J. Multidrug efflux pumps in gram-negative bacteria and their role in antibiotic resistance. Future Microbiol. 2014, 9, 1165-1177. [CrossRef]

27. Choi, U.; Lee, C.R. Distinct roles of outer membrane porins in antibiotic resistance and membrane integrity in Escherichia coli. Front Microbiol. 2019, 10, 953. [CrossRef]

28. Cui, L.; Ma, X.; Sato, K.; Okuma, K.; Tenover, F.C.; Mamizuka, E.M.; Gemmell, C.G.; Kim, M.N.; Ploy, M.C.; El-Solh, N.; et al. Cell wall thickening is a common feature of vancomycin resistance in Staphylococcus aureus. J. Clin. Microbiol. 2003, 41, 5-14.

29. Garcia, A.B.; Vinuela-Prieto, J.M.; Lopez-Gonzalez, L.; Candel, F.J. Correlation between resistance mechanisms in Staphylococcus aureus and cell wall and septum thickening. Infect. Drug Resist 2017, 10, 353-356. [CrossRef]

30. Bebear, C.; Pereyre, S.; Peuchant, O. Mycoplasma pneumoniae: Susceptibility and resistance to antibiotics. Future Microbiol. 2011, 6, 423-431. [CrossRef]

31. Tooke, C.L.; Hinchliffe, P.; Bragginton, E.C.; Colenso, C.K.; Hirvonen, V.H.A.; Takebayashi, Y.; Spencer, J. Beta-lactamases and beta-lactamase inhibitors in the 21st century. J. Mol. Biol. 2019, 431, 3472-3500. [CrossRef]

32. Casciaro, B.; Loffredo, M.R.; Luca, V.; Verrusio, W.; Cacciafesta, M.; Mangoni, M.L. Esculentin-1a derived antipseudomonal peptides: Limited induction of resistance and synergy with aztreonam. Protein Pept. Lett. 2018, 25, 1155-1162. [CrossRef] [PubMed]

33. Jeong, J.H.; Cha, H.J.; Kim, Y.G. Crystal structures of penicillin-binding protein d2 from Listeria monocytogenes and structural basis for antibiotic specificity. Antimicrob. Agents Chemother. 2018, 62, e00796-18. [CrossRef] [PubMed]

34. Cong, Y.; Yang, S.; Rao, X. Vancomycin resistant Staphylococcus aureus infections: A review of case updating and clinical features. J. Adv. Res. 2020, 21, 169-176. [CrossRef] [PubMed]

35. Hassanzadeh, S.; Ganjloo, S.; Pourmand, M.R.; Mashhadi, R.; Ghazvini, K. Epidemiology of efflux pumps genes mediating resistance among Staphylococcus aureus; a systematic review. Microb. Pathog. 2020, 139, 103850. [CrossRef] [PubMed]

36. Ghajavand,H.; Kargarpour Kamakoli, M.; Khanipour, S.; Pourazar Dizaji, S.; Masoumi, M.; Rahimi Jamnani, F.; Fateh, A.; Yaseri, M.; Siadat, S.D.; Vaziri, F. Scrutinizing the drug resistance mechanism of multi- and extensively-drug resistant Mycobacterium tuberculosis: Mutations versus efflux pumps. Antimicrob. Resist Infect. Control 2019, 8, 70. [CrossRef] [PubMed]

37. Miryala, S.K.; Anbarasu, A.; Ramaiah, S. Systems biology studies in Pseudomonas aeruginosa PA01 to understand their role in biofilm formation and multidrug efflux pumps. Microb. Pathog. 2019, 136, 103668. [CrossRef]

38. Casciaro, B.; Dutta, D.; Loffredo, M.R.; Marcheggiani, S.; McDermott, A.M.; Willcox, M.D.; Mangoni, M.L. Esculentin-1a derived peptides kill Pseudomonas aeruginosa biofilm on soft contact lenses and retain antibacterial activity upon immobilization to the lens surface. Pept. Sci. 2018, 110, e23074. [CrossRef]

39. Crouzet, M.; Claverol, S.; Lomenech, A.M.; Le Senechal, C.; Costaglioli, P.; Barthe, C.; Garbay, B.; Bonneu, M.; Vilain, S. Pseudomonas aeruginosa cells attached to a surface display a typical proteome early as $20 \mathrm{~min}$ of incubation. PLoS ONE 2017, 12, e0180341. [CrossRef]

40. Oliveira, W.F.; Silva, P.M.S.; Silva, R.C.S.; Silva, G.M.M.; Machado, G.; Coelho, L.; Correia, M.T.S. Staphylococcus aureus and Staphylococcus epidermidis infections on implants. J. Hosp. Infect. 2018, 98, 111-117. [CrossRef]

41. Casciaro, B.; Moros, M.; Rivera-Fernandez, S.; Bellelli, A.; de la Fuente, J.M.; Mangoni, M.L. Gold-nanoparticles coated with the antimicrobial peptide esculentin-1a(1-21) $\mathrm{NH}_{2}$ as a reliable strategy for antipseudomonal drugs. Acta. Biomater. 2017, 47, 170-181. [CrossRef] 
42. d'Angelo, I.; Casciaro, B.; Miro, A.; Quaglia, F.; Mangoni, M.L.; Ungaro, F. Overcoming barriers in Pseudomonas aeruginosa lung infections: Engineered nanoparticles for local delivery of a cationic antimicrobial peptide. Coll. Surf. B Biointerfaces 2015, 135, 717-725. [CrossRef] [PubMed]

43. Casciaro, B.; d'Angelo, I.; Zhang, X.; Loffredo, M.R.; Conte, G.; Cappiello, F.; Quaglia, F.; Di, Y.P.; Ungaro, F.; Mangoni, M.L. Poly(lactide- co-glycolide) nanoparticles for prolonged therapeutic efficacy of esculentin-1a-derived antimicrobial peptides against Pseudomonas aeruginosa lung infection: In vitro and in vivo studies. Biomacromolecules 2019, 20, 1876-1888. [CrossRef] [PubMed]

44. Lopez, Y.; Soto, S.M. The usefulness of microalgae compounds for preventing biofilm infections. Antibiotics 2019, 9, 9. [CrossRef] [PubMed]

45. Casciaro, B.; Lin, Q.; Afonin, S.; Loffredo, M.R.; de Turris, V.; Middel, V.; Ulrich, A.S.; Di, Y.P.; Mangoni, M.L. Inhibition of Pseudomonas aeruginosa biofilm formation and expression of virulence genes by selective epimerization in the peptide esculentin-1a(1-21) $\mathrm{NH}_{2}$. FEBS J. 2019, 286, 3874-3891. [CrossRef] [PubMed]

46. Fitzgerald, M.; Heinrich, M.; Booker, A. Medicinal plant analysis: A historical and regional discussion of emergent complex techniques. Front Pharmacol. 2019, 10, 1480. [CrossRef]

47. Sneader, W. The prehistoric period. In Drug Discovery; John Wiley \& Sons: Chichester, UK; Hoboken, NJ, USA, 2006; pp. 6-11.

48. Sneader, W. Pre-hellenic civilisations. In Drug discovery; John Wiley \& Sons: Chichester, UK; Hoboken, NJ, USA, 2006; pp. 12-17.

49. Cragg, G.M.; Newman, D.J. Natural products: A continuing source of novel drug leads. Biochim. Biophys. Acta. 2013, 1830, 3670-3695. [CrossRef]

50. Eigenschink, M.; Dearing, L.; Dablander, T.E.; Maier, J.; Sitte, H.H. A critical examination of the main premises of traditional chinese medicine. Wien. Klin. Wochenschr. 2020, 132, 260-273. [CrossRef]

51. Sneader, W. Drug Discovery; John Wiley and Sons: Chichester, UK; Hoboken, NJ, USA, 2006; pp. 18-23.

52. Sneader, W. Herbals. In Drug Discovery; John Wiley and Sons: Chichester, UK; Hoboken, NJ, USA, 2006; pp. 32-40.

53. Calixto, J.B. The role of natural products in modern drug discovery. An. Acad. Bras. Cienc. 2019, 91, e20190105. [CrossRef]

54. Palermo, R.; Ghirga, F.; Piccioni, M.G.; Bernardi, F.; Zhdanovskaya, N.; Infante, P.; Mori, M. Natural products inspired modulators of cancer stem cells-specific signaling pathways notch and hedgehog. Curr. Pharm. Des. 2018, 24, 4251-4269. [CrossRef]

55. Romano, J.D.; Tatonetti, N.P. Informatics and computational methods in natural product drug discovery: A review and perspectives. Front. Genet 2019, 10, 368. [CrossRef]

56. David, B.; Wolfender, J.-L.; Dias, D.A. The pharmaceutical industry and natural products: Historical status and new trends. Phytochem. Rev. 2015, 14, 299-315. [CrossRef]

57. Bucar, F.; Wube, A.; Schmid, M. Natural product isolation-how to get from biological material to pure compounds. Nat. Prod. Rep. 2013, 30, 525-545. [CrossRef] [PubMed]

58. Kinghorn, A.D.; Pan, L.; Fletcher, J.N.; Chai, H. The relevance of higher plants in lead compound discovery programs. J. Nat. Prod. 2011, 74, 1539-1555. [CrossRef] [PubMed]

59. Henrich, C.J.; Beutler, J.A. Matching the power of high throughput screening to the chemical diversity of natural products. Nat. Prod. Rep. 2013, 30, 1284-1298. [CrossRef]

60. Coan, K.E.; Ottl, J.; Klumpp, M. Non-stoichiometric inhibition in biochemical high-throughput screening. Expert Opin. Drug Discov. 2011, 6, 405-417. [CrossRef]

61. Johnson, T.A.; Sohn, J.; Inman, W.D.; Estee, S.A.; Loveridge, S.T.; Vervoort, H.C.; Tenney, K.; Liu, J.; Ang, K.K.; Ratnam, J.; et al. Natural product libraries to accelerate the high-throughput discovery of therapeutic leads. J. Nat. Prod. 2011, 74, 2545-2555. [CrossRef]

62. Zhu, J.; Ven, W.; Verbiest, T.; Koeckelberghs, G.; Chen, C.; Cui, Y.; Vermorken, A. Polyphenols can inhibit furin in vitro as a result of the reactivity of their auto-oxidation products to proteins. Curr. Med. Chem. 2012, 20, 840-850.

63. Balunas, M.J.; Su, B.; Landini, S.; Brueggemeier, R.W.; Kinghorn, A.D. Interference by naturally occurring fatty acids in a noncellular enzyme-based aromatase bioassay. J. Nat. Prod. 2006, 69, 700-703. [CrossRef]

64. Corson, T.W.; Crews, C.M. Molecular understanding and modern application of traditional medicines: Triumphs and trials. Cell 2007, 130, 769-774. [CrossRef]

65. Heinrich, M. Ethnopharmacology in the 21st century-Grand challenges. Front Pharmacol. 2010, 1, 8. [CrossRef] 
66. Heinrich, M.; Gibbons, S. Ethnopharmacology in drug discovery: An analysis of its role and potential contribution. J. Pharm. Pharmacol. 2001, 53, 425-432. [CrossRef] [PubMed]

67. Wright, G.D. Unlocking the potential of natural products in drug discovery. Microb. Biotechnol. 2019, 12, 55-57. [CrossRef]

68. Linington, R.G.; Kubanek, J.; Luesch, H. New methods for isolation and structure determination of natural products. Nat. Prod. Rep. 2019, 36, 942-943. [CrossRef]

69. Wilson, B.A.P.; Thornburg, C.C.; Henrich, C.J.; Grkovic, T.; O'Keefe, B.R. Creating and screening natural product libraries. Nat. Prod. Rep. 2020. [CrossRef] [PubMed]

70. Infante, P.; Mori, M.; Alfonsi, R.; Ghirga, F.; Aiello, F.; Toscano, S.; Ingallina, C.; Siler, M.; Cucchi, D.; Po, A.; et al. Gli1/DNA interaction is a druggable target for hedgehog-dependent tumors. EMBO J. 2015, 34, 200-217. [CrossRef] [PubMed]

71. Ingallina, C.; Costa, P.M.; Ghirga, F.; Klippstein, R.; Wang, J.T.; Berardozzi, S.; Hodgins, N.; Infante, P.; Pollard, S.M.; Botta, B.; et al. Polymeric glabrescione b nanocapsules for passive targeting of hedgehogdependent tumor therapy. In Vitro Nanomed. (Lond.) 2017, 12, 711-728. [CrossRef] [PubMed]

72. Mori, M.; Tottone, L.; Quaglio, D.; Zhdanovskaya, N.; Ingallina, C.; Fusto, M.; Ghirga, F.; Peruzzi, G.; Crestoni, M.E.; Simeoni, F.; et al. Identification of a novel chalcone derivative that inhibits notch signaling in t-cell acute lymphoblastic leukemia. Sci. Rep. 2017, 7, 2213. [CrossRef]

73. Casciaro, B.; Calcaterra, A.; Cappiello, F.; Mori, M.; Loffredo, M.R.; Ghirga, F.; Mangoni, M.L.; Botta, B.; Quaglio, D. Nigritanine as a new potential antimicrobial alkaloid for the treatment of Staphylococcus aureusinduced infections. Toxins 2019, 11, 511. [CrossRef]

74. Cevatemre, B.; Botta, B.; Mori, M.; Berardozzi, S.; Ingallina, C.; Ulukaya, E. The plant-derived triterpenoid tingenin $\mathrm{b}$ is a potent anticancer agent due to its cytotoxic activity on cancer stem cells of breast cancer in vitro. Chem. Biol. Interact. 2016, 260, 248-255. [CrossRef] [PubMed]

75. Circi, S.; Capitani, D.; Randazzo, A.; Ingallina, C.; Mannina, L.; Sobolev, A.P. Panel test and chemical analyses of commercial olive oils: A comparative study. Chem. Biol. Technol. Agric. 2017, 4, 18. [CrossRef]

76. Berardozzi, S.; Bernardi, F.; Infante, P.; Ingallina, C.; Toscano, S.; De Paolis, E.; Alfonsi, R.; Caimano, M.; Botta, B.; Mori, M.; et al. Synergistic inhibition of the hedgehog pathway by newly designed smo and gli antagonists bearing the isoflavone scaffold. Eur. J. Med. Chem. 2018, 156, 554-562. [CrossRef] [PubMed]

77. Quaglio, D.; Zhdanovskaya, N.; Tobajas, G.; Cuartas, V.; Balducci, S.; Christodoulou, M.S.; Fabrizi, G.; Gargantilla, M.; Priego, E.M.; Carmona Pestana, A.; et al. Chalcones and chalcone-mimetic derivatives as notch inhibitors in a model of $\mathrm{t}-\mathrm{cell}$ acute lymphoblastic leukemia. ACS Med. Chem. Lett. 2019, 10, 639-643. [CrossRef] [PubMed]

78. Lospinoso Severini, L.; Quaglio, D.; Basili, I.; Ghirga, F.; Bufalieri, F.; Caimano, M.; Balducci, S.; Moretti, M.; Romeo, I.; Loricchio, E.; et al. A smo/gli multitarget hedgehog pathway inhibitor impairs tumor growth. Cancers 2019, 11, 1518. [CrossRef] [PubMed]

79. Mushtaq, S.; Abbasi, B.H.; Uzair, B.; Abbasi, R. Natural products as reservoirs of novel therapeutic agents. EXCLI J. 2018, 17, 420-451. [PubMed]

80. Atanasov, A.G.; Waltenberger, B.; Pferschy-Wenzig, E.M.; Linder, T.; Wawrosch, C.; Uhrin, P.; Temml, V.; Wang, L.; Schwaiger, S.; Heiss, E.H.; et al. Discovery and resupply of pharmacologically active plant-derived natural products: A review. Biotechnol. Adv. 2015, 33, 1582-1614. [CrossRef] [PubMed]

81. Newman, D.J.; Cragg, G.M. Natural products as sources of new drugs over the nearly four decades from 01/1981 to 09/2019. J. Nat. Prod. 2020, 83, 770-803. [CrossRef]

82. Osbourn, A.E.; Lanzotti, V. Plant-Derived Natural Products; Springer: Dordrecht, The Netherlands, 2009; pp. 361-384.

83. Subramani, R.; Narayanasamy, M.; Feussner, K.D. Plant-derived antimicrobials to fight against multi-drugresistant human pathogens. 3 Biotech. 2017, 7, 172. [CrossRef]

84. Lei, Y.; Fu, P.; Jun, X.; Cheng, P. Pharmacological properties of geraniol-A review. Planta Med. 2019, 85, 48-55. [CrossRef]

85. Cragg, G.; Newman, D.; Kingston, D. Terrestrial plants as a source of novel pharmaceutical agents. Compr. Nat. Prod. II Chem. Biol. 2010, 2, 5-39.

86. Vieira, A.J.; Beserra, F.P.; Souza, M.C.; Totti, B.M.; Rozza, A.L. Limonene: Aroma of innovation in health and disease. Chem. Biol. Interact. 2018, 283, 97-106. [CrossRef] [PubMed] 
87. Butler, M.S.; Robertson, A.A.; Cooper, M.A. Natural product and natural product derived drugs in clinical trials. Nat. Prod. Rep. 2014, 31, 1612-1661. [CrossRef] [PubMed]

88. Wozniak, L.; Skapska, S.; Marszalek, K. Ursolic acid-A pentacyclic triterpenoid with a wide spectrum of pharmacological activities. Molecules 2015, 20, 20614-20641. [CrossRef] [PubMed]

89. Anand, U.; Jacobo-Herrera, N.; Altemimi, A.; Lakhssassi, N. A comprehensive review on medicinal plants as antimicrobial therapeutics: Potential avenues of biocompatible drug discovery. Metabolites 2019, 9, 258. [CrossRef] [PubMed]

90. Ludwiczuk, A.; Skalicka-Woźniak, K.; Georgiev, M. Terpenoids. In Pharmacognosy; Elsevier: Amsterdam, The Netherlands, 2017; pp. 233-266.

91. Ashour, M.; Wink, M.; Gershenzon, J. Biochemistry of terpenoids: Monoterpenes, sesquiterpenes and diterpenes. Annu. Plant Rev. 2018, 40, 258-303.

92. Guimaraes, A.C.; Meireles, L.M.; Lemos, M.F.; Guimaraes, M.C.C.; Endringer, D.C.; Fronza, M.; Scherer, R. Antibacterial activity of terpenes and terpenoids present in essential oils. Molecules 2019, 24, 2471. [CrossRef]

93. Jiang, K.; Chen, L.L.; Wang, S.F.; Wang, Y.; Li, Y.; Gao, K. Anti-inflammatory terpenoids from the leaves and twigs of Dysoxylum gotadhora. J. Nat. Prod. 2015, 78, 1037-1044. [CrossRef]

94. Nothias-Scaglia, L.F.; Pannecouque, C.; Renucci, F.; Delang, L.; Neyts, J.; Roussi, F.; Costa, J.; Leyssen, P.; Litaudon, M.; Paolini, J. Antiviral activity of diterpene esters on chikungunya virus and hiv replication. J. Nat. Prod. 2015, 78, 1277-1283. [CrossRef]

95. Alqahtani, A.; Hamid, K.; Kam, A.; Wong, K.H.; Abdelhak, Z.; Razmovski-Naumovski, V.; Chan, K.; Li, K.; Groundwater, P.; Li, G. The pentacyclic triterpenoids in herbal medicines and their pharmacological activities in diabetes and diabetic complications. Curr. Med. Chem. 2012, 20, 908-931.

96. Yuan, X.L.; Mao, X.X.; Du, Y.M.; Yan, P.Z.; Hou, X.D.; Zhang, Z.F. Anti-tumor activity of cembranoid-type diterpenes isolated from Nicotiana tabacum L. Biomoleculars 2019, 9, 45. [CrossRef]

97. Quaglio, D.; Corradi, S.; Erazo, S.; Vergine, V.; Berardozzi, S.; Sciubba, F.; Cappiello, F.; Crestoni, M.E.; Ascenzioni, F.; Imperi, F.; et al. Structural elucidation and antimicrobial characterization of novel diterpenoids from Fabiana densa var. Ramulosa. ACS Med. Chem. Lett. 2020, 11, 760-765. [CrossRef] [PubMed]

98. Imane, N.I.; Fouzia, H.; Azzahra, L.F.; Ahmed, E.; Ismail, G.; Idrissa, D.; Mohamed, K.-H.; Sirine, F.; L'Houcine, O.; Noureddine, B. Chemical composition, antibacterial and antioxidant activities of some essential oils against multidrug resistant bacteria. Eur. J. Integr. Med. 2020, 35, 101074. [CrossRef]

99. Langeveld, W.T.; Veldhuizen, E.J.; Burt, S.A. Synergy between essential oil components and antibiotics: A review. Crit. Rev. Microbiol. 2014, 40, 76-94. [CrossRef] [PubMed]

100. Yap, P.S.; Lim, S.H.; Hu, C.P.; Yiap, B.C. Combination of essential oils and antibiotics reduce antibiotic resistance in plasmid-conferred multidrug resistant bacteria. Phytomedicine 2013, 20, 710-713. [CrossRef] [PubMed]

101. Thimmappa, R.; Geisler, K.; Louveau, T.; O'Maille, P.; Osbourn, A. Triterpene biosynthesis in plants. Annu. Rev. Plant Biol. 2014, 65, 225-257. [CrossRef]

102. Demetzos, C.; Dimas, K. Labdane-type diterpenes: Chemistry and biological activity. Stud. Nat. Prod. Chem. 2001, 25, 235-292.

103. Taylor, R.S.L.; Towers, G.H.N. Antibacterial constituents of the nepalese medicinal herb, Centipeda Minima. Phytochemistry 1998, 47, 631-634. [CrossRef]

104. Kawazoe, K.; Tsubouchi, Y.; Abdullah, N.; Takaishi, Y.; Shibata, H.; Higuti, T.; Hori, H.; Ogawa, M. Sesquiterpenoids from Artemisia gilvescens and an anti-mrsa compound. J. Nat. Prod. 2003, 66, 538-539. [CrossRef]

105. Ordonez, P.E.; Quave, C.L.; Reynolds, W.F.; Varughese, K.I.; Berry, B.; Breen, P.J.; Malagon, O.; Vidari, G.; Smeltzer, M.S.; Compadre, C.M. Sesquiterpene lactones from Gynoxys verrucosa and their anti-mrsa activity. J. Ethnopharmacol. 2011, 137, 1055-1059. [CrossRef]

106. Mustaffa, F.; Indurkar, J.; Ismail, S.; Shah, M.; Mansor, S.M. An antimicrobial compound isolated from Cinnamomum iners leaves with activity against methicillin-resistant Staphylococcus aureus. Molecules 2011, 16, 3037-3047. [CrossRef]

107. Corlay, N.; Lecso-Bornet, M.; Leborgne, E.; Blanchard, F.; Cachet, X.; Bignon, J.; Roussi, F.; Butel, M.J.; Awang, K.; Litaudon, M. Antibacterial labdane diterpenoids from Vitex vestita. J. Nat. Prod. 2015, 78, 1348-1356. [CrossRef] 
108. Farimani, M.M.; Taleghani, A.; Aliabadi, A.; Aliahmadi, A.; Esmaeili, M.A.; Namazi Sarvestani, N.; Khavasi, H.R.; Smiesko, M.; Hamburger, M.; Nejad Ebrahimi, S. Labdane diterpenoids from Salvia leriifolia: Absolute configuration, antimicrobial and cytotoxic activities. Planta Med. 2016, 82, 1279-1285. [CrossRef] [PubMed]

109. Qiao, Y.; Liu, Y.; Duan, X.; Chen, C.; Liu, J.; Zhu, H.; Xue, Y.; Zhang, Y. A pair of epimeric cassane-type diterpenoids and a new labdane-type derivative from Caesalpinia decapetala. Tetrahedron 2018, 74, 3852-3857. [CrossRef]

110. Siddique, H.; Pendry, B.; Rahman, M.M. Terpenes from Zingiber montanum and their screening against multi-drug resistant and methicillin resistant Staphylococcus aureus. Molecules 2019, 24, 385. [CrossRef] [PubMed]

111. Gaspar-Marques, C.; Rijo, P.; Simoes, M.F.; Duarte, M.A.; Rodriguez, B. Abietanes from Plectranthus grandidentatus and P. hereroensis against methicillin- and vancomycin-resistant bacteria. Phytomedicine 2006, 13, 267-271. [CrossRef]

112. Jurkaninova, S.; Kubinova, R.; Nejezchlebova, M.; Gazdova, M.; Hanakova, Z.; Dall'Acqua, S. Anti-mrsa activity of abietane diterpenes from Coleus blumei benth. Nat. Prod. Res. 2019, 1-7. [CrossRef]

113. Porto, T.S.; Simao, M.R.; Carlos, L.Z.; Martins, C.H.; Furtado, N.A.; Said, S.; Arakawa, N.S.; dos Santos, R.A.; Veneziani, R.C.; Ambrosio, S.R. Pimarane-type diterpenes obtained by biotransformation: Antimicrobial properties against clinically isolated gram-positive multidrug-resistant bacteria. Phytother. Res. 2013, 27, 1502-1507. [CrossRef]

114. Stavri, M.; Paton, A.; Skelton, B.W.; Gibbons, S. Antibacterial diterpenes from Plectranthus ernstii. J. Nat. Prod. 2009, 72, 1191-1194. [CrossRef]

115. Smith, E.; Williamson, E.; Zloh, M.; Gibbons, S. Isopimaric acid from Pinus nigra shows activity against multidrug-resistant and emrsa strains of Staphylococcus aureus. Phytother. Res. 2005, 19, 538-542. [CrossRef]

116. Soares, A.C.F.; Matos, P.M.; Silva, K.F.d.; Martins, C.H.G.; Veneziani, R.C.S.; Ambrósio, S.R.; Dias, H.J.; Santos, R.A.d.; Heleno, V.C.G. Antimicrobial potential of natural and semi-synthetic ent-kaurane and ent-pimarane diterpenes against clinically isolated gram-positive multidrug-resistant bacteria. J. Braz. Chem. Soc. 2019, 30, 333-341. [CrossRef]

117. Pfeifer Barbosa, A.L.; Wenzel-Storjohann, A.; Barbosa, J.D.; Zidorn, C.; Peifer, C.; Tasdemir, D.; Cicek, S.S. Antimicrobial and cytotoxic effects of the Copaifera reticulata oleoresin and its main diterpene acids. J. Ethnopharmacol. 2019, 233, 94-100. [CrossRef]

118. Ghirga, F.S.R.; Cavinato, L.; Lo Sciuto, A.; Corradi, S.; Quaglio, D.; Calcaterra, A.; Casciaro, B.; Loffredo, M.R.; Cappiello, F.; Morelli, P.; et al. A novel colistin adjuvant identified by virtual screening for arnt inhibitors. J. Antimicrob. Chem. 2020. [CrossRef] [PubMed]

119. Pooi Yin, C.; Navaratnam, P.; Chung, L.Y. Synergistic antimicrobial activity between pentacyclic triterpenoids and antibiotics against Staphylococcus aureus strains. Annals Clin. Microbial. Antimicrob. 2011, 10, 25.

120. Horiuchi, K.; Shiota, S.; Hatano, T.; Yoshida, T.; Kuroda, T.; Tsuchiya, T. Antimicrobial activity of oleanolic acid from Salvia officinalis and related compounds on vancomycin-resistant enterococci (vre). Biol. Pharm. Bull. 2007, 30, 1147-1149. [CrossRef] [PubMed]

121. Dwivedi, G.R.; Maurya, A.; Yadav, D.K.; Khan, F.; Darokar, M.P.; Srivastava, S.K. Drug resistance reversal potential of ursolic acid derivatives against nalidixic acid- and multidrug-resistant Escherichia. coli. Chem. Biol. Drug Des. 2015, 86, 272-283. [CrossRef] [PubMed]

122. Wang, C.M.; Chen, H.T.; Wu, Z.Y.; Jhan, Y.L.; Shyu, C.L.; Chou, C.H. Antibacterial and synergistic activity of pentacyclic triterpenoids isolated from Alstonia scholaris. Molecules 2016, 21, 139. [CrossRef] [PubMed]

123. Cruz, B.G.; Rodrigues Teixeira, A.M.; Silva, P.T.D.; Vasconcelos Evaristo, F.F.; de Vaconcelos, M.A.; Holanda Teixeira, E.; Dos Santos, H.S.; Bandeira, P.N.; Sena-Junior, D.M.; Barreto, V.P.; et al. Antimicrobial activity of the lupane triterpene 3beta,6beta,16beta-trihydroxylup-20(29)-ene isolated from Combretum leprosum mart. J. Med. Microbiol. 2019, 68, 1438-1444. [CrossRef]

124. Gutierrez-Lugo, M.T.; Singh, M.P.; Maiese, W.M.; Timmermann, B.N. New antimicrobial cycloartane triterpenes from Acalypha communis. J. Nat. Prod. 2002, 65, 872-875. [CrossRef]

125. Wang, X.Y.; Tang, G.H.; Yuan, C.M.; Zhang, Y.; Zou, T.; Yu, C.; Zhao, Q.; Hao, X.J.; He, H.P. Aphagrandinoids a-d, cycloartane triterpenoids with antibacterial activities from Aphanamix. grandifolia. Fitoterapia 2013, 85, 64-68. [CrossRef]

126. Mosa, R.; Mandlakayise, L.; Thandeka, V.; Andy, R. Antibacterial activity of two triterpenes from stem bark of Protorhus longifolia. J. Med. Plant. Res. 2014, 8, 686-702. 
127. Kim, C.; Hesek, D.; Lee, M.; Mobashery, S. Potentiation of the activity of beta-lactam antibiotics by farnesol and its derivatives. Bioorg. Med. Chem. Lett. 2018, 28, 642-645. [CrossRef]

128. Gupta, V.K.; Verma, S.; Pal, A.; Srivastava, S.K.; Srivastava, P.K.; Darokar, M.P. In vivo efficacy and synergistic interaction of 16alpha-hydroxycleroda-3, 13 (14) z-dien-15, 16-olide, a clerodane diterpene from Polyalthia longifolia against methicillin-resistant Staphylococcus saureus. Appl. Microbiol. Biotechnol. 2013, 97, 9121-9131. [CrossRef] [PubMed]

129. Sayout, A.; Ouarhach, A.; Rabie, R.; Dilagui, I.; Soraa, N.; Romane, A. Evaluation of antibacterial activity of lavandula pedunculata subsp. atlantica (braun-blanq.) romo essential oil and selected terpenoids against resistant bacteria strains-structure-activity relationships. Chem. Biodivers. 2020, 17, e1900496. [CrossRef] [PubMed]

130. Lorenzi, V.; Muselli, A.; Bernardini, A.F.; Berti, L.; Pages, J.M.; Amaral, L.; Bolla, J.M. Geraniol restores antibiotic activities against multidrug-resistant isolates from gram-negative species. Antimicrob. Agents Chemother. 2009, 53, 2209-2211. [CrossRef] [PubMed]

131. de Breij, A.; Karnaoukh, T.G.; Schrumpf, J.; Hiemstra, P.S.; Nibbering, P.H.; van Dissel, J.T.; de Visser, P.C. The licorice pentacyclic triterpenoid component 18beta-glycyrrhetinic acid enhances the activity of antibiotics against strains of methicillin-resistant Staphylococcus aureus. Eur. J. Clin. Microbiol. Infect. Dis. 2016, 35, 555-562. [CrossRef] [PubMed]

132. Reynolds, W.F.; Enriquez, R.G. Terpenes: Mono-, sesqui-, and higher Terpenes. In Modern NMR Approaches to the Structure Elucidation of Natural Products: Volume 2: Data Acquisition and Applications to Compound Classes; The Royal Society of Chemistry: Cambridge, UK, 2017.

133. Griffin, S.G.; Wyllie, S.G.; Markham, J.L.; Leach, D.N. The role of structure and molecular properties of terpenoids in determining their antimicrobial activity. Flavour Fragr. J. 1999, 14, 322-332. [CrossRef]

134. Mahizan, N.; Yang, S.K.; Moo, C.-L.; Song, A.; Chong, C.M.; Chong, C.; Abushelaibi, A.; Lim, E.; Lai, K.-S. Terpene derivatives as a potential agent against antimicrobial resistance (amr) pathogens. Molecules 2019, 24, 2631. [CrossRef]

135. Sayout, A.; Ouarhach, A.; Dilagui, I.; Soraa, N.; Romane, A. Antibacterial activity and chemical composition of essential oil from Lavandula tenuisecta coss.Ex ball. An endemic species from Morocco. Eur. J. Integrative Med. 2020, 33, 101017. [CrossRef]

136. Brilhante, R.S.; Valente, L.G.; Rocha, M.F.; Bandeira, T.J.; Cordeiro, R.A.; Lima, R.A.; Leite, J.J.; Ribeiro, J.F.; Pereira, J.F.; Castelo-Branco, D.S.; et al. Sesquiterpene farnesol contributes to increased susceptibility to beta-lactams in strains of Burkholderia pseudomallei. Antimicrob. Agents Chemother. 2012, 56, 2198-2200. [CrossRef]

137. Kuo-Hsiung, L.; Ibuka, T.; Rong-Yang, W.; Geissman, T.A. Structure-antimicrobial activity relationships among the sesquiterpene lactones and related compounds. Phytochemistry 1977, 16, 1177-1181. [CrossRef]

138. Gibbons, S. Anti-staphylococcal plant natural products. Nat. Prod. Rep. 2004, 21, 263-277. [CrossRef]

139. Gonçalves, O.; Pereira, R.; Gonçalves, F.; Mendo, S.; Coimbra, M.A.; Rocha, S.M. Evaluation of the mutagenicity of sesquiterpenic compounds and their influence on the susceptibility towards antibiotics of two clinically relevant bacterial strains. Mutat. Res./Genet. Toxicol. Environ. Mutagen. 2011, 723, 18-25. [CrossRef] [PubMed]

140. Veneziani, R.; Ambrosio, S.; Martins, C.H.; Lemes, D.C.; Oliveira, L.C. Antibacterial potential of diterpenoids. Stud. Nat. Prod. Chem. 2017, 54, 109-139.

141. Gupta, V.K.; Tiwari, N.; Gupta, P.; Verma, S.; Pal, A.; Srivastava, S.K.; Darokar, M.P. A clerodane diterpene from Polyalthia longifolia as a modifying agent of the resistance of methicillin resistant Staphylococcus aureus. Phytomedicine 2016, 23, 654-661. [CrossRef] [PubMed]

142. Neto, Í.; Faustino, C.; Rijo, P. Antimicrobial abietane diterpenoids against resistant bacteria and biofilms. In The Battle Against Microbial Pathogens: Basic Science, Technological Advances and Educational Programs; Méndez-Vilas, A., Ed.; Formatex Research Center: Badajoz, Spain, 2015; Volume 1, pp. 15-26.

143. Urzua, A.; Rezende, M.C.; Mascayano, C.; Vasquez, L. A structure-activity study of antibacterial diterpenoids. Molecules 2008, 13, 882-891. [CrossRef]

144. Zloh, M.; Kaatz, G.W.; Gibbons, S. Inhibitors of multidrug resistance (mdr) have affinity for mdr substrates. Bioorg. Med. Chem. Lett. 2004, 14, 881-885. [CrossRef]

145. Imperi, F.; Ascenzioni, F.; Mori, M.; Ghirga, F.; Quaglio, D.; Corradi, S.; Lo Sciuto, A.; Botta, B.; Calcaterra, A.; Stefanelli, R. Inibitori della antibiotico-resistenza mediata da ArnT. IT102019000012888, 2019. Available online: https://www.uniroma1.it/it/brevetto/102019000012888 (accessed on 15 May 2020).

146. Chung, P.Y. Novel targets of pentacyclic triterpenoids in Staphylococcus aureus: A systematic review. Phytomedicine 2019, 73, 152933. [CrossRef] 
147. Catteau, L.; Zhu, L.; Van Bambeke, F.; Quetin-Leclercq, J. Natural and hemi-synthetic pentacyclic triterpenes as antimicrobials and resistance modifying agents against Staphylococcus aureus: A review. Phytochem. Rev. 2018, 17, 1129-1163. [CrossRef]

148. Paul, S.; Talla, E.; Matchawe, C.; Alembert, T.T.; Elisabeth, Z.M.; Sophie, L.; Luce, V.E.; Maurice, T.F.; Joël, Y.; Alex, A.; et al. Pentacyclic triterpenes and crude extracts with antimicrobial activity from Cameroonian brown propolis samples. J. Appl. Pharm. Sci. 2014, 4, 1-9.

(c) (

(C) 2020 by the authors. Licensee MDPI, Basel, Switzerland. This article is an open access article distributed under the terms and conditions of the Creative Commons Attribution (CC BY) license (http://creativecommons.org/licenses/by/4.0/). 